\title{
Akt and SHP-1 are DC-intrinsic checkpoints for tumor immunity
}

\author{
Yaron Carmi, ${ }^{1,2}$ Tyler R. Prestwood, ${ }^{1,3}$ Matthew H. Spitzer, ${ }^{4}$ Ian L. Linde, ${ }^{1,5}$ Jonathan Chabon, ${ }^{6}$ \\ Nathan E. Reticker-Flynn, ${ }^{1}$ Nupur Bhattacharya, ${ }^{1}$ Hong Zhang, ${ }^{1}$ Xiangyue Zhang, ${ }^{1}$ Pamela A. Basto, ${ }^{1}$ \\ Bryan M. Burt, ${ }^{7}$ Michael N. Alonso, ${ }^{1}$ and Edgar G. Engleman ${ }^{1}$ \\ 'Department of Pathology, Stanford University School of Medicine, Stanford, California, USA. 'Department of Pathology, \\ The Sackler School of Medicine, Tel-Aviv University, Ramat Aviv, Israel. ${ }^{3}$ Program in Stem Cell Biology and Regenerative \\ Medicine, Stanford University School of Medicine, Stanford, California, USA. ${ }^{4}$ Department of Microbiology and Immunology, \\ University of California, San Francisco, San Francisco, California, USA. ${ }^{5}$ Program in Immunology, Stanford University School \\ of Medicine, Stanford, California, USA. ${ }^{6}$ Center for Epigenetics Research, Memorial Sloan Kettering Cancer Center, New York, \\ New York, USA. ${ }^{7}$ Division of General Thoracic Surgery, Baylor College of Medicine, Houston, Texas, USA.
}

BM-derived DC (BMDC) are powerful antigen-presenting cells. When loaded with immune complexes (IC), consisting of tumor antigens bound to antitumor antibody, BMDC induce powerful antitumor immunity in mice. However, attempts to employ this strategy clinically with either tumor-associated DC (TADC) or monocyte-derived DC (MoDC) have been disappointing. To investigate the basis for this phenomenon, we compared the response of BMDC, TADC, and MODC to tumor IgG-IC. Our findings revealed, in both mice and humans, that upon exposure to IgC-IC, BMDC internalized the IC, increased costimulatory molecule expression, and stimulated autologous $\mathrm{T}$ cells. In contrast, TADC and, surprisingly, MoDC remained inert upon contact with IC due to dysfunctional signaling following engagement of Fc $\gamma$ receptors. Such dysfunction is associated with elevated levels of the Src homology region 2 domain-containing phosphatase-1 (SHP-1) and phosphatases regulating Akt activation. Indeed, concomitant inhibition of both SHP-1 and phosphatases that regulate Akt activation conferred upon TADC and MoDC the capacity to take up and process IC and induce antitumor immunity in vivo. This work identifies the molecular checkpoints that govern activation of MoDC and TADC and their capacity to elicit T cell immunity.

Authorship note: Y. Carmi and T.R. Prestwood contributed equally to this work.

Conflict of interest: The authors have declared that no conflict of interest exists.

Submitted: June 8, 2016 Accepted: September 26, 2016 Published: November 3, 2016

Reference information: JCI Insight. 2016;1(18):e89020. doi:10.1172/jci.insight.89020.

\section{Introduction}

Progressively growing tumors emerge from a unique environment, which results from interactions among tumor cells, their surrounding stroma, and infiltrating cells of the host immune system (1-4). Over the past two decades, major efforts have aimed at analyzing and identifying these tumor-infiltrating immune cells and distinguishing between their protumor and antitumor activities (5-10). Recently, a number of studies have established a strong correlation between tumor-associated Th1 signatures and survival of cancer patients $(11,12$, and reviewed in ref. 13). Moreover, the recent success of $\mathrm{T}$ cell-mediated therapies has made clear the clinical benefit of harnessing this effector arm to attack cancer cells (14-17). However, activation of an effective $\mathrm{T}$ cell response requires presentation of antigens in a stimulatory context by DC. Such antigen presentation is dependent on the ability of DC to internalize the antigens through receptors associated with a danger signal, usually by scavenger or pattern-recognition receptors (18-21). Recently, we have demonstrated that tumor-binding IgG antibodies can load DC with tumor antigens and initiate a $\mathrm{T}$ cell-mediated immune response, preventing tumor recurrence and eradicating established tumors (22).

There are four IgG subclasses (IgG1, IgG2a/c, IgG2b and IgG3 in mice, and IgG1 to IgG4 in humans), which differ in the nature of the antigens they recognize, their affinity for various Fc $\gamma$ receptors (Fc $\gamma \mathrm{Rs})$, and the immunological processes they induce (23-26). Ligation of the activating receptors, Fc $\gamma$ RIII and Fc $\gamma$ RIV, or the high-affinity Fc $\gamma$ RI on DC results in phosphorylation of tyrosine protein kinase SYK (Syk) and activation of the MAPK pathway. In contrast, signaling through the inhibitory receptor Fc $\gamma$ RII leads to phosphorylation of its immunoreceptor tyrosine-based inhibitory motif (ITIM) and recruitment of Src homology region 2 domain-containing phosphatase-1 (SHP-1), which blocks calcium influx and MAPK signaling (23-26). In a classic study, Nimmerjahn and Ravetch showed that engagement of the Fc $\gamma$ RIV by 
IgG2a/c promotes potent antitumor immunity, while signaling through Fc $\gamma$ RIII is much less effective (27).

Previous studies have shown that DC loaded with immune complexes (IC) can induce a strong antitumor immune response in a number of mouse models. Rafiq et al. showed that ovalbumin-IC taken up by $\mathrm{DC}$ can be subsequently presented to ovalbumin-specific $\mathrm{CD} 4^{+}$and $\mathrm{CD} 8^{+} \mathrm{T}$ cells to induce their proliferation (28). Similarly, Schuurhuis et al. demonstrated that immunization with DC loaded with ovalbumin-IC elicits superior protection against ovalbumin-expressing tumors compared with other immune activating strategies (29). Importantly, however, these results were all achieved with BM-derived DC (BMDC), and attempts to employ this understanding to activate tumor-associated DC (TADC) with tumor-reactive antibodies have shown little to no efficacy. We recently showed that, in contrast to BMDC, TADC cannot respond to IC alone, but rather require additional signals to take up IC (22). Nonetheless, the mechanism that prevents their activation remains unclear. Here, we identified the molecules that regulate and prevent their activation.

Our findings show that the unresponsiveness of TADC to IC does not result from the tumor microenvironment, but rather reflects a more basic control mechanism. In both mice and humans, BMDC were strikingly superior to TADC and peripheral blood monocyte-derived DC (MoDC) of tumor-free donors in their capacity to become activated and induce $\mathrm{CD} 4^{+} \mathrm{T}$ cell proliferation following incubation with allogeneic IgG-tumor cell IC (alloIgG-IC). High intrinsic phosphatase activity prevented the activation of downstream signaling pathways in these unresponsive DC. We further found that once monocytes are released from their BM niche into the circulation, they markedly elevate the levels of phosphorylated SHP-1 as well as phosphatases that regulate Akt activation. These results provide insights into fundamental control mechanisms in DC biology that can be targeted to enhance cancer immunotherapy.

\section{Results}

AlloIgG-IC-loaded BMDC, but not TADC or MoDC, prevent tumor recurrence following resection. In a recent study, we found that administration of alloIgG prior to tumor challenge can protect the host and that these antibodies are ineffective against established tumors since TADC cannot process IC (22). We therefore sought to test the capacity of alloIgG to prevent tumor recurrence in the adjuvant setting (i.e., after surgical resection of the tumor). B16F10 melanoma or LMP pancreatic tumor cells were injected s.c. into mice above the right flank and allowed to grow until tumors reached $20-25 \mathrm{~mm}^{2}$ in size. Tumors were then surgically removed from animals, leaving margins of about $1 \mathrm{~mm}$, and enzymatically digested to obtain a single-cell suspension. Tumor cells were enriched by depleting leukocytes using anti-CD45 antibody-conjugated magnetic beads. Tumor cells were then fixed and coated with alloIgG to form IC, which were incubated overnight with BMDC. Tumor-resected mice were injected s.c. with either BMDC loaded with these IC (alloIgG-IC), alloIgG-IC alone, or with purified alloIgG alone (Figure 1A). Administration of alloIgG into tumor-resected animals was ineffective, and the rate of tumor regrowth in these mice was comparable to that seen in untreated mice. Injection of alloIgG-IC had only a minor effect on tumor recurrence, with $20 \%$ of mice experiencing significant protection (Figure 1B). In contrast, most of the mice that were injected with BMDC loaded with alloIgG-IC remained tumor free throughout the experiment, which was terminated at 80 days (Figure 1B).

We next tested whether the monocytes and DC subsets capable of infiltrating resection sites could protect against tumor recurrence. As illustrated in Figure 1C, monocytes from the blood ( $\mathrm{FSC}^{\mathrm{lo}} / \mathrm{SSC}^{\mathrm{lo}} / \mathrm{Ly} 6 \mathrm{C}^{+} /$ $\left.\mathrm{CD}_{115^{+}}\right)$and $\mathrm{BM}\left(\mathrm{FSC}^{\mathrm{lo}} / \mathrm{SSC}^{\mathrm{lo}} / \mathrm{Ly}_{6} \mathrm{C}^{+} / \mathrm{CD} 115^{+}\right)$of tumor-bearing mice were isolated and cultured in granulocyte-macrophage CSF (GM-CSF) to obtain DC. TADC (FSC ${ }^{\mathrm{lo}} / \mathrm{SSC}^{\mathrm{lo}} / \mathrm{Ly} 6 \mathrm{C}^{\text {neg }} / \mathrm{CD} 11 \mathrm{c}^{+} / \mathrm{MHC}$ $\mathrm{II}^{\mathrm{hi}}$ ) were used immediately following isolation (Supplemental Figure 1A; supplemental material available online with this article; doi:10.1172/jci.insight.89020DS1). Culture of sorted BM monocytes yielded $>90 \%$ pure populations of MHC II ${ }^{\text {int }} / \mathrm{CD} 11 \mathrm{~b}^{\text {hi }} / \mathrm{CD} 135^{\text {neg }}$ cells (Supplemental Figure $1 \mathrm{~B}$ ), which resemble the macrophage/monocyte population described by Helft et al. (30). Injection of blood MoDC or TADC loaded with alloIgG-IC into the tumor-resected mice from which they were derived failed to protect against tumor recurrence (Figure 1D). Taken together, these results demonstrate a unique capability of BMDC to process alloIgG-IC and thereby protect mice from tumor recurrence.

AlloIgG-IC-loaded BMDC induce T cell immunity, which prevents tumor recurrence. We next sought to determine if the inability of blood MoDC and TADC to prevent tumor recurrence results from systemic immune suppression induced by the tumors or reflects the normal function of these cell types. Therefore, patrolling monocytes $\left(\mathrm{FSC}^{\mathrm{lo}} / \mathrm{SSC}^{\mathrm{lo}} / \mathrm{Ly} 6 \mathrm{C}^{\mathrm{neg}} / \mathrm{CD} 115^{+}\right)$and inflammatory monocytes $\left(\mathrm{FSC}^{\mathrm{lo}} / \mathrm{SSC}^{\mathrm{lo}} / \mathrm{Ly} 6 \mathrm{C}^{+} / \mathrm{CD} 115^{+}\right)$ 
A

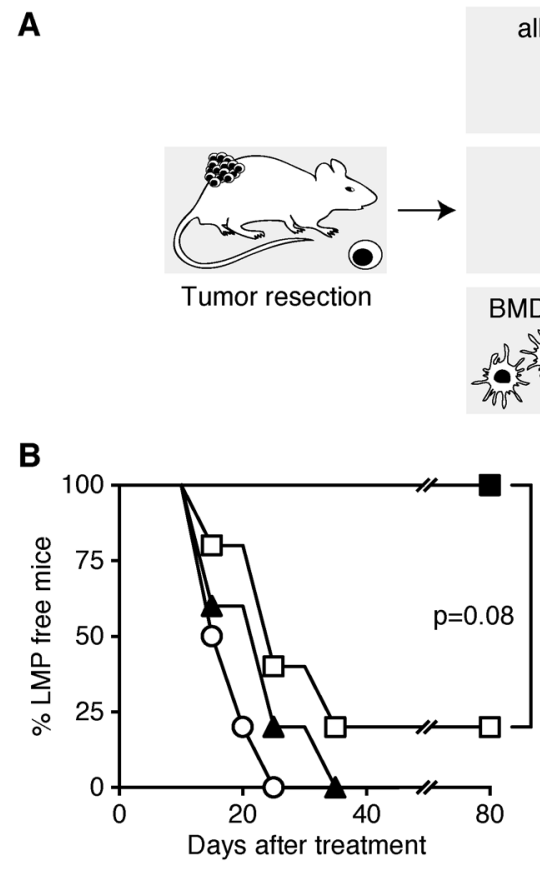

allogeneic $\lg G$
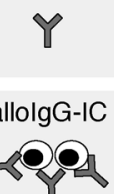

MDC + allolgG-IC
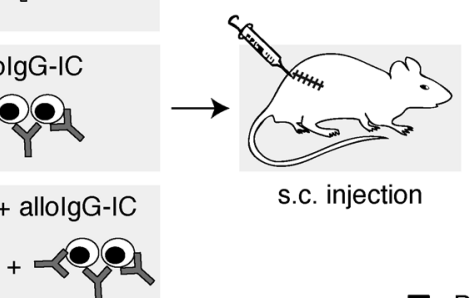

s.c. injection

C

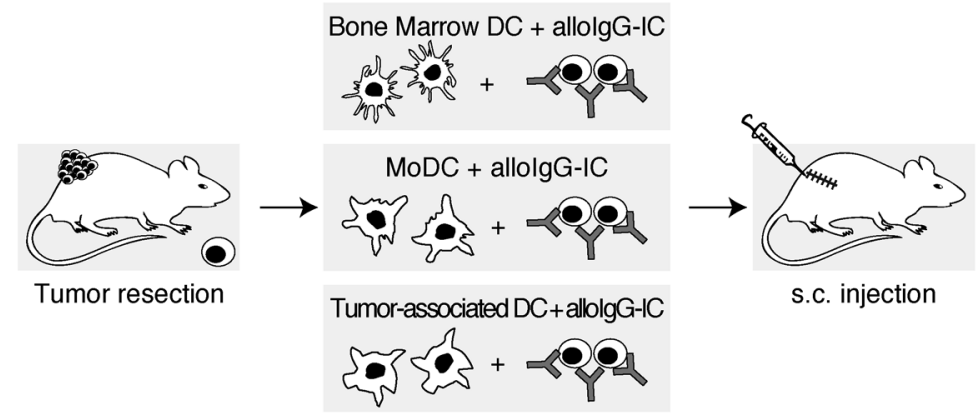

D
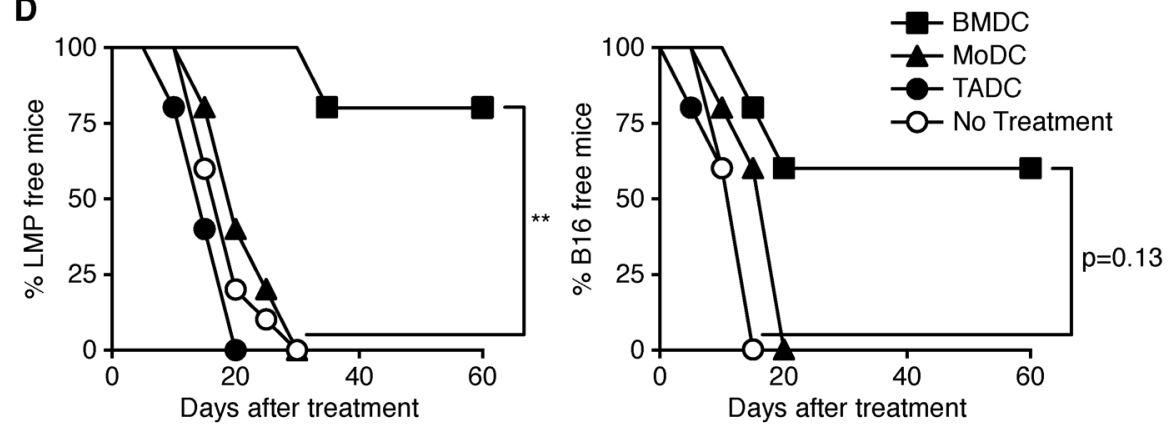

Figure 1. AllolgG-IC-loaded BMDC, but not TADC or MoDC, prevent tumor recurrence following resection. (A) Experimental design: Tumor-resected mice were left untreated or were injected s.c. with $0.5 \mathrm{mg}$ allogenic IgG, with allogeneic IgG-tumor cell immune complexes (allolgG-IC), or with BM-derived DC (BMDC) activated with allolgG-IC. (B) The rate of tumor recurrence in LMP and B16 tumor-resected mice following treatment. (C) Experimental design: Tumor-resected mice were left untreated or were injected s.c. with BMDC, blood monocyte-derived DC (MoDC), or tumor-associated DC (TADC), each isolated from tumor-bearing mice, preactivated with corresponding tumor cells and allolgG-IC. (D). The rates of tumor recurrence in untreated tumor-resected mice or mice injected with various DC populations. Shown is 1 representative experiment of 4 independent experiments performed ( $n=10$ for control group and $n=5$ in each treatment). Significance was determined by the log-rank Mantel-Cox test using Bonferroni-adjusted $P$ values. ${ }^{*} P \leq 0.01$.

from blood as well as BM monocytes were isolated from naive syngeneic mice and cultured with GM-CSF to obtain DC. These DC subsets were then incubated overnight with alloIgG-IC, washed extensively, and injected into the corresponding tumor-resected mice (illustrated in Figure 2A). Consistent with our results using blood MoDC from tumor-bearing mice, alloIgG-IC-loaded MoDC from naive mice failed to provide protection, as the rate of tumor recurrence was comparable to that in untreated mice or mice treated with TADC (Figure 2B). In contrast, most mice injected with alloIgG-IC-loaded BMDC were protected from tumor recurrence (Figure $2 \mathrm{~B}$ ).

The hallmark of DC is their remarkable ability to activate and promote $\mathrm{T}$ cell proliferation. We therefore compared the capacity of each DC subset to skew T cells toward a Th1 phenotype and support their

proliferation. Each DC subset was incubated overnight with alloIgG-IC, washed extensively, and incubated with syngeneic splenic CD $4^{+} \mathrm{T}$ cells (illustrated in Figure $2 \mathrm{C}$ ). As a reference point, we used bulk spleen DC, which exhibit a well-documented capacity to present extrinsic antigens in this assay. The bulk spleen DC consisted mainly of $\mathrm{CD}_{11} \mathrm{~b}^{+}$myeloid DC (about $80 \%$ ), but also CD4-/CD $8 \alpha^{-}$and CD $8 \alpha^{+}$DC (Supplemental Figure 1C). After 6 days, $\mathrm{CD}^{+} \mathrm{T}$ cell proliferation rates were analyzed based on the incorporation of radio-labeled thymidine, and the amount of IFN- $\gamma$ secreted into the supernatants was also measured. Consistent with their poor protection from tumor recurrence in vivo, both MoDC and TADC incubated with alloIgG-IC could not induce $\mathrm{CD}^{+} \mathrm{T}$ cell proliferation or significant IFN- $\gamma$ secretion. In contrast, identically treated BMDC, and to a lesser extent splenic DC, induced marked $\mathrm{CD}^{+} \mathrm{T}$ cell proliferation and IFN- $\gamma$ secretion (Figure 2, D and E). The capacity of DC preincubated with alloIgG-IC to support CD8 ${ }^{+} \mathrm{T}$ cell proliferation was also tested. Consistent with their poor activation of $\mathrm{CD}^{+} \mathrm{T}$ cells, TADC and MoDC 
A

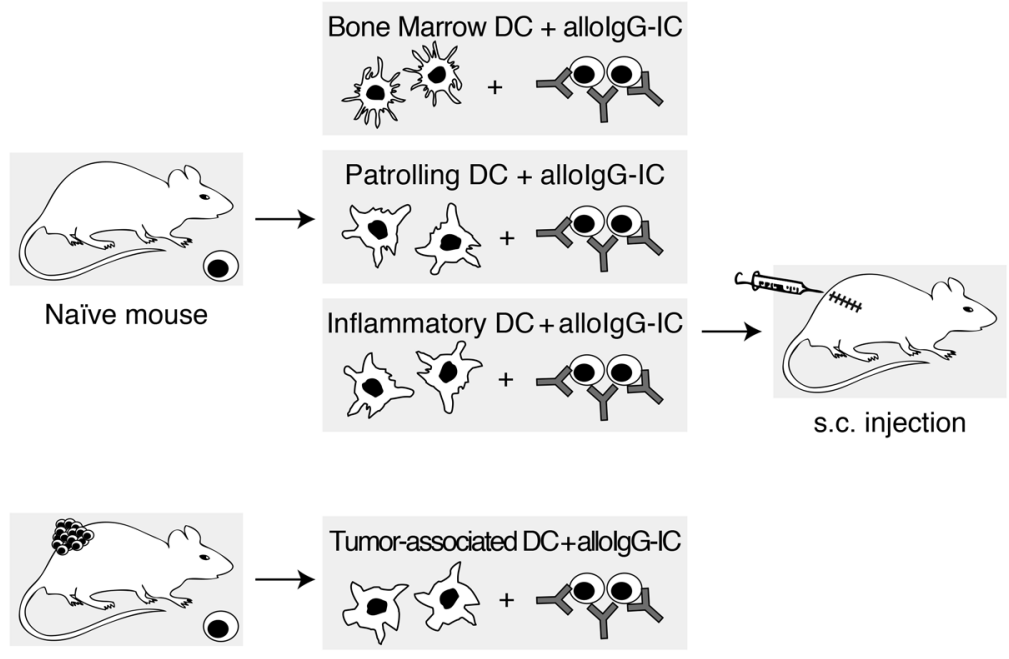

Tumor resection

C

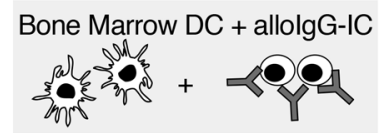

Spleen DC + allolgG-IC

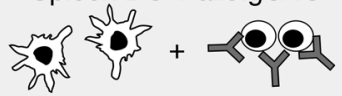

Patrolling DC + allolgG-IC

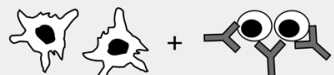

Inflammatory DC + allolgG-IC

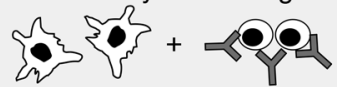

Tumor-associated DC+allolgG-IC

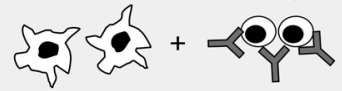

B
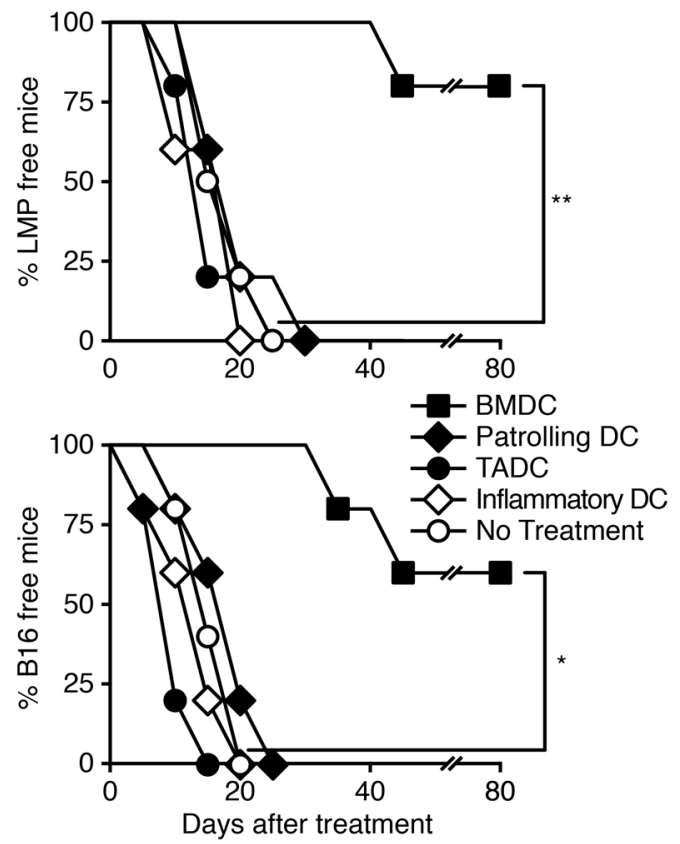

CD4 ${ }^{+} \mathrm{T}$ cell proliferation
D

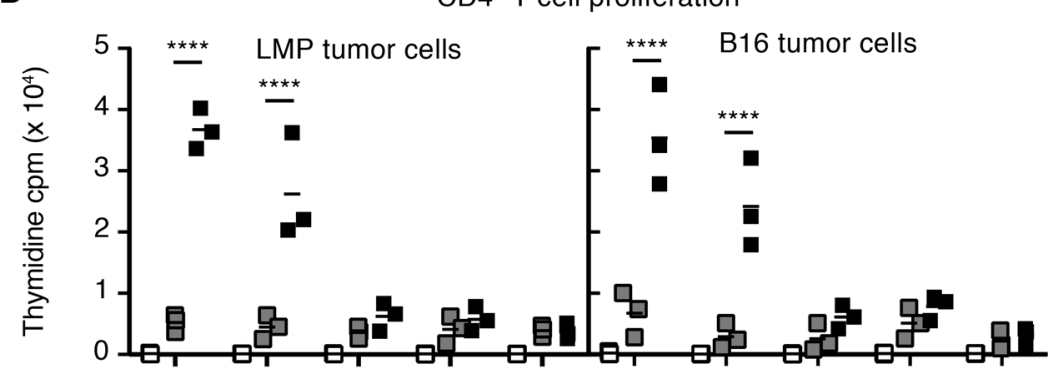

E

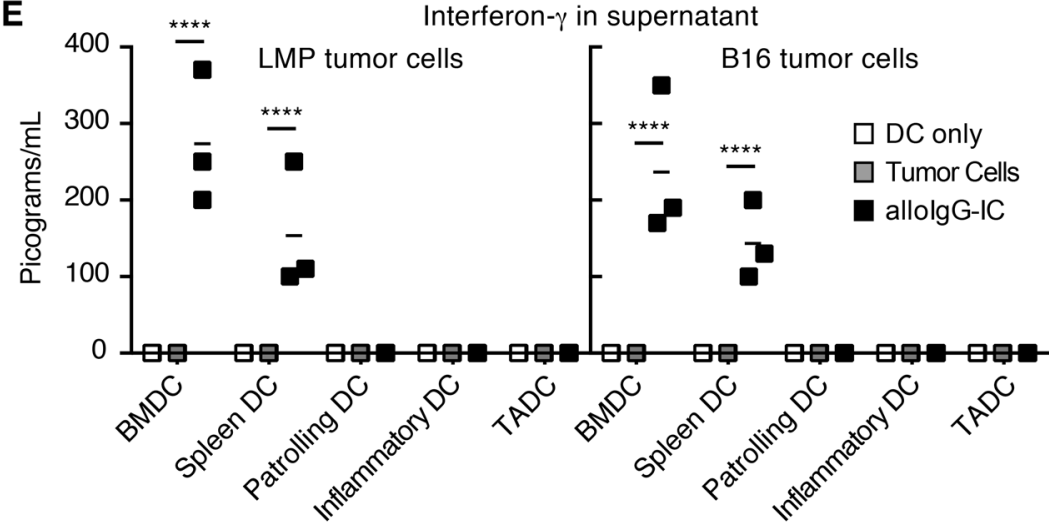

Figure 2. AllolgG-IC-loaded BMDC induce T cell immunity to prevent tumor recurrence. (A) Experimental design: Tumor-resected mice were left untreated or were injected s.c. with DC subsets from naive mice preactivated with corresponding tumor cells bound with allogeneic lgG in immune complexes (allolgG-IC). (B) The rates of tumor recurrence in untreated tumor-resected mice or in mice injected with BM-derived DC (BMDC), patrolling blood monocyte-derived DC (Patrolling DC), inflammatory blood monocyte-derived DC (Inflammatory DC), or tumor-associated DC (TADC) preactivated with allolgG-IC. Significance was determined by the log-rank Mantel-Cox test using Bonferroni-adjusted $P$ values. (C) Experimental design: Sorted DC subsets were activated with allolgG-IC overnight, washed, and incubated with splenic CD4+ ${ }^{+}$cells at a 1:5 ratio for an additional 6 to 7 days. (D) T cell proliferation and (E) IFN- $\gamma$ secretion following incubation with allolgG-IC-activated DC. Shown is 1 representative experiment of 4 independent experiments performed ( $n=3$ in each treatment). Statistical significance was determined by 2-way ANOVA with Tukey's multiple comparisons test. ${ }^{*} P<0.05,{ }^{* *} P<0.01,{ }^{* * *} P<0.0001$. 
A

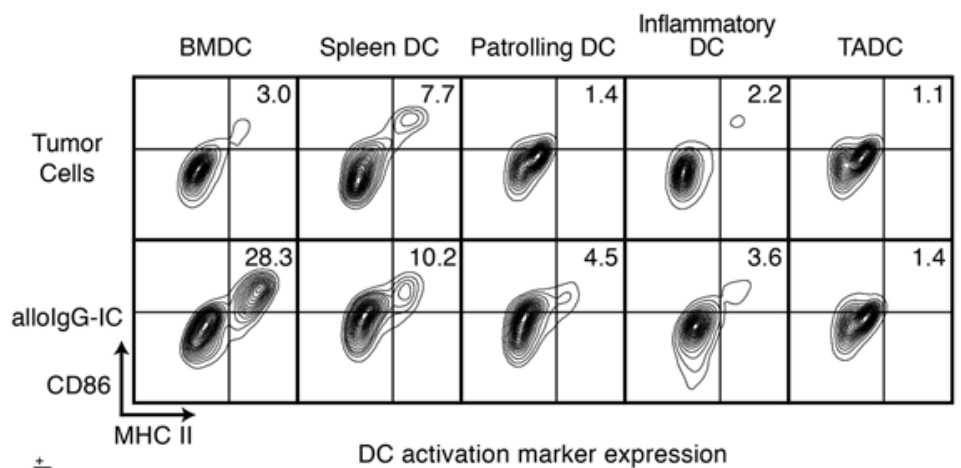

B
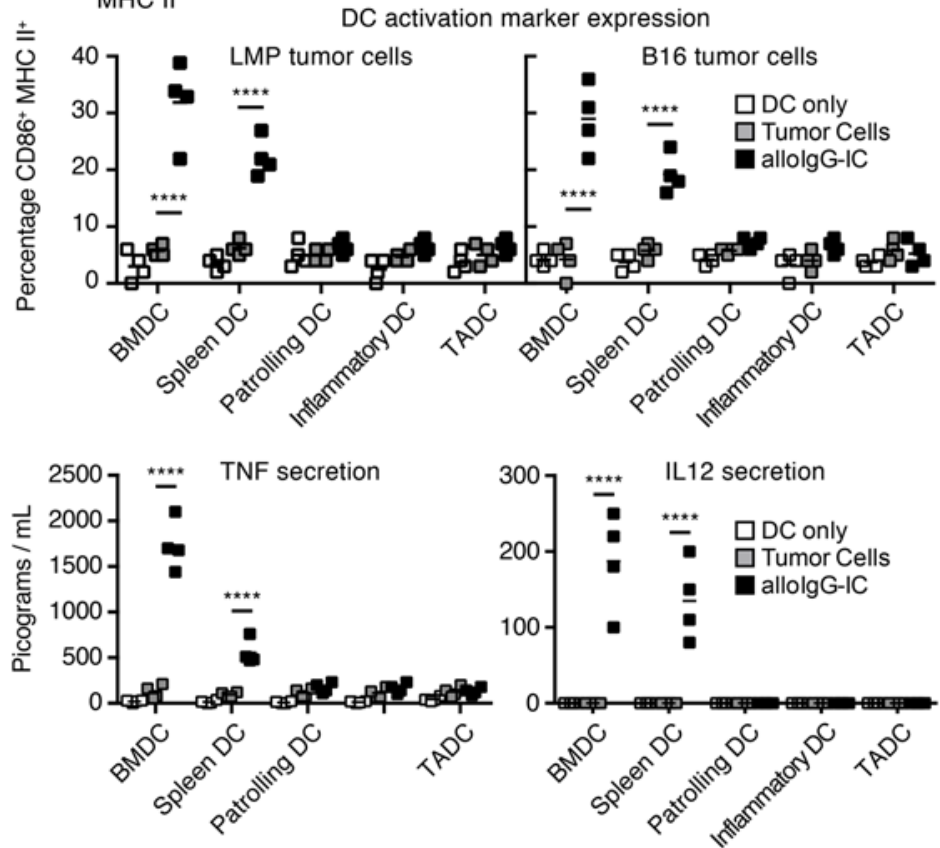

C

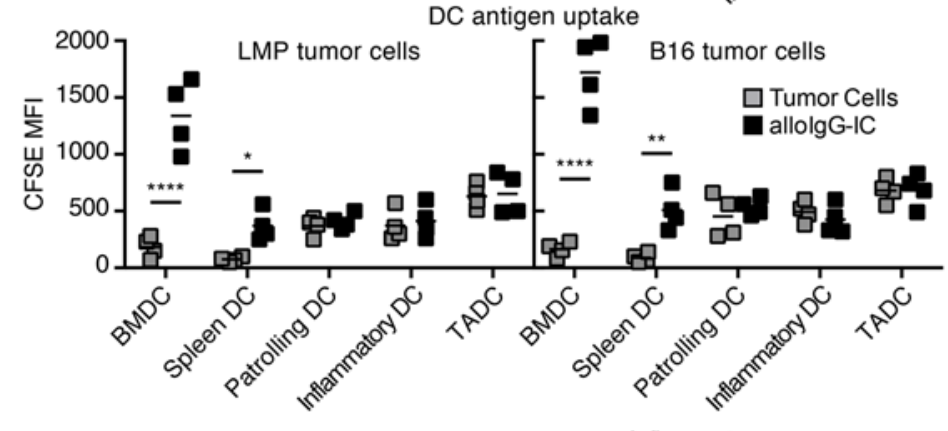

D

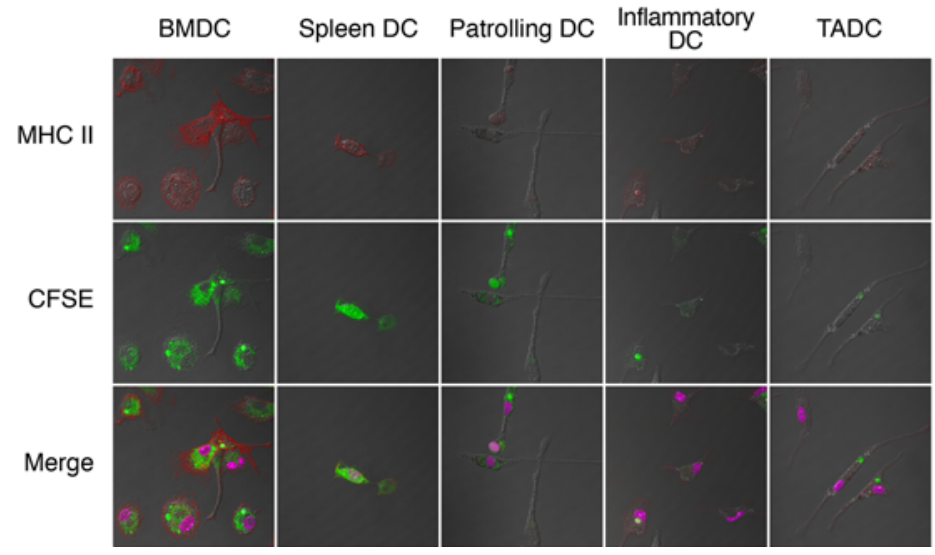

Figure 3. BMDC exhibit a unique ability to be directly activated by allolgG-IC. (A) Flow cytometry analysis of MHC II and CD86 expression on BM monocyte-derived DC (BMDC), patrolling blood monocyte-derived DC (Patrolling $D C$ ), inflammatory blood monocyte-derived DC (Inflammatory $D C$ ), or tumor-associated DC (TADC) following overnight incubation with tumor cells or allogeneic IgG-tumor cell immune complexes (allolgG-IC). Graphs show the percentages of DC expressing both MHC II and CD86. Statistical significance was determined by 2-way ANOVA with Tukey's multiple comparisons test. (B) Levels of TNF and IL-12 in the supernatants of $\mathrm{DC}$ subsets incubated overnight with LMP tumor cells or allogeneic C57BL/6 IgG-LMP tumor cell immune complexes (allolgG-IC). Statistical significance was determined by 2-way ANOVA with Tukey adjustment for multiple comparisons. (C) Flow cytometric analysis of uptake of CFSE-labeled tumor cells or allolgG-IC by DC after 14 hours of incubation. Statistical significance was determined by 2-way ANOVA with Sidak's multiple comparisons test. (D) Immunofluorescence microscopy of tumor antigen uptake (green) and MHC II expression (red) by DC populations following overnight incubation with LMP tumor cells or allogeneic C57BL/6 IgG-LMP tumor cell immune complexes (allolgG-IC). Original magnification, $\times 40$. Shown are representative photomicrographs from 1 experiment of 5 performed. Graphs show the mean levels of 1 representative experiment of 3 independently repeated experiments. ${ }^{*} P<0.05,{ }^{*} P<0.01,{ }^{* * * *} P<0.0001$.

did not support $C D 8^{+} \mathrm{T}$ cell proliferation. Interestingly, BMDC induced only marginal $\mathrm{CD} 8^{+}$proliferation, while spleen DC induced significant, yet low levels of $\mathrm{CD}^{+}$proliferation (Supplemental Figure 2). Taken together, it seems that $\mathrm{CD} 4^{+} \mathrm{T}$ cells, rather than $\mathrm{CD} 8^{+}$ $\mathrm{T}$ cells, are the primary target cells for myeloid $\mathrm{DC}$ following activation with IC.

$B M D C$ exhibit a unique ability to be directly activated by alloIg $G$-IC. Since the activation of T cells depends on the ability of DC to internalize and present antigens in a stimulatory context, we next compared these properties in different DC subsets following incubation with alloIgG-IC. Inflammatory and patrolling monocytes from the blood as well as BM monocytes were isolated from naive mice and cultured for 4 days to obtain DC. TADC were used immediately after their isolation. The capacity of these DC to respond to alloIgG-IC was compared with that of DC freshly isolated from the spleens of naive mice. DC subsets were then cultured alone, with fixed tumor cells, or with fixed tumor cell-IC. BMDC, and to a lesser extent splenic DC, showed significant activation following exposure to IC, as manifested by increases in CD86 and MHC II expression (Figure 3A) and secretion of IL-12 and TNF (Figure 3B). In contrast, activation of inflammatory MoDC, patrolling MoDC, or TADC resulted in only a marginal increase in CD86 and MHC II levels and low levels of IL-12 and TNF following culture with alloIgG-IC (Figure 3, A and B). We next compared the ability of these DC to inter- 
A
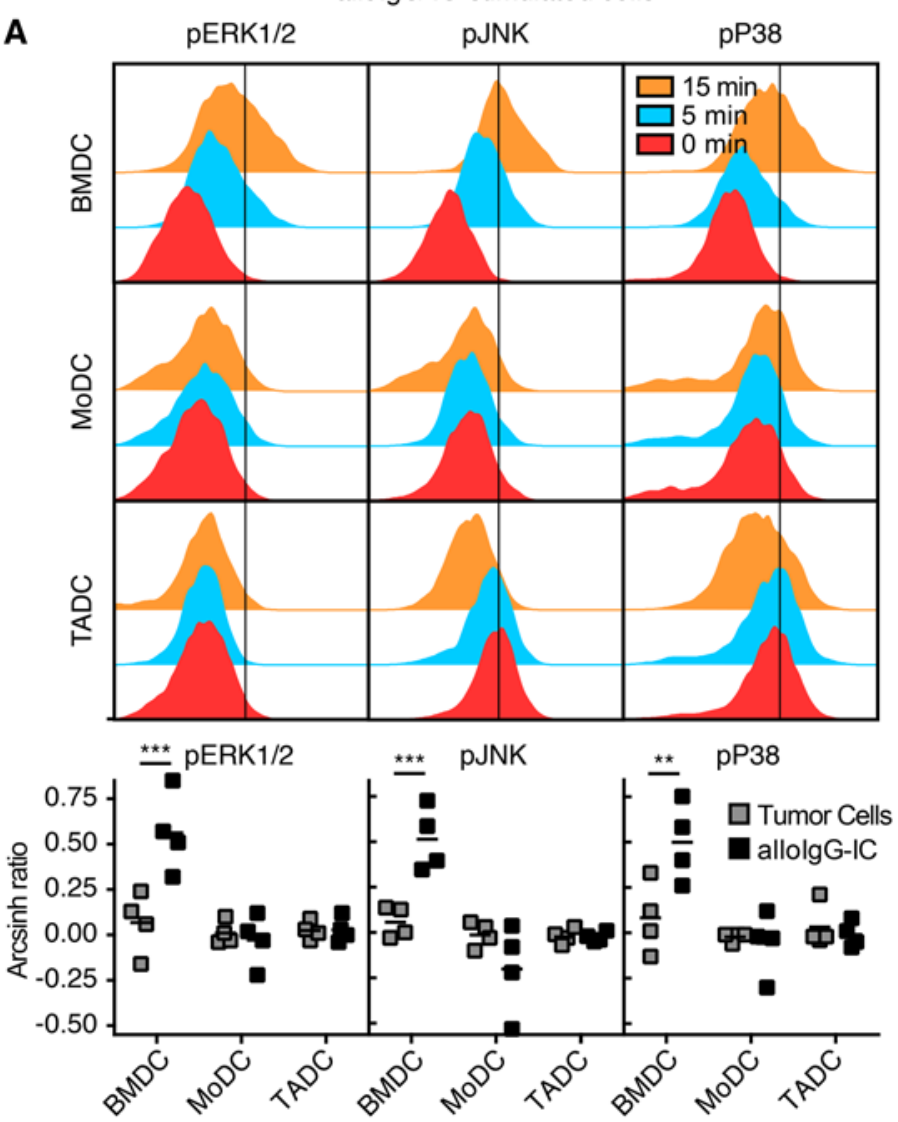

B

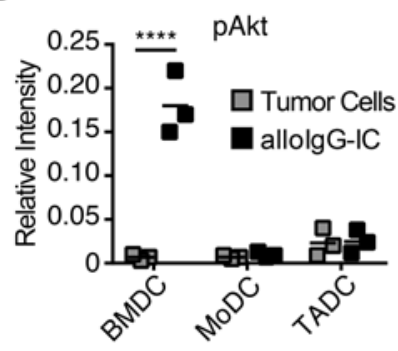

C allolgG-IC-stimulated cells

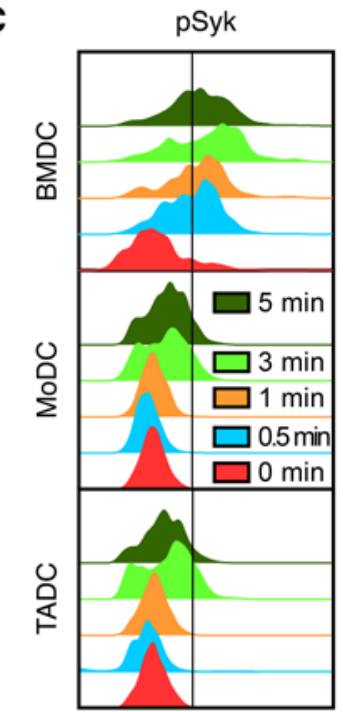

pSyk

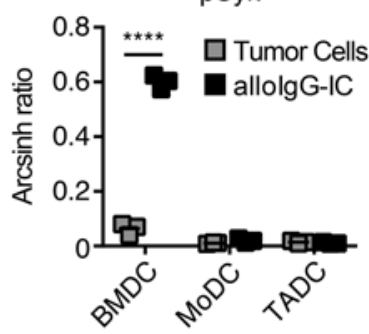

Figure 4. Phosphorylation of Syk after Fc $\gamma \mathbf{R}$ ligation is inhibited in TADC and MoDC. (A) Histograms show phosphorylated p38 (pP38), pERK1/2, and pJNK levels in BM-derived DC (BMDC), blood monocyte-derived DC (MoDC), and tumor-associated DC (TADC) incubated with tumor cells or with C57BL/6 allogeneic IgG-LMP tumor cell immune complexes (allolgG-IC) for 0, 5, and 15 minutes. Graphs show arcsinh ratios of phospho-species in DC populations after 15-minute incubation with allolgG-IC over baseline levels from tumor cell-stimulated DC $(n=4)$. (B) pAkt levels in DC incubated with tumor cells or allolgG-IC, as measured by PathScan Intracellular signaling array $(n=3)$. Graphs show the pixel density of pAkt in activated DC. (C) Histograms show pSyk levels in DC incubated with allolgG-IC. Graphs show arcsinh ratios of pSyk in DC incubated for 1 minute with LMP cells or allolgG-IC over baseline levels obtained from unstimulated DC $(n=3)$. Mean levels from 1 representative experiment of at least 3 independently repeated experiments are shown. Statistical significance was determined by 2-way ANOVA with Sidak's multiple comparisons test. ${ }^{* *} P<0.01,{ }^{* *} P<0.001,{ }^{* * * *} P<0.0001$.

nalize tumor-derived proteins. Fixed tumor cells were stained with carboxyfluorescein succinimidyl ester (CFSE) and added to DC cultures before or after coating the tumor cells with alloIgG to form alloIgG-IC. Following overnight incubation, DC were extensively washed and their tumor uptake was analyzed by flow cytometry and confocal microscopy. Consistent with the activation state of each population, BMDC, and to a lesser extent spleen DC, internalized significant levels of tumor-derived antigen, while the other DC populations did not (Figure 3, C and D). Similar patterns of DC activation were observed following incubation with freeze-thawed lysates or with live tumor cells coated with alloIgG (data not shown). Taken together, these results suggest that the inability of peripheral MoDC to protect mice and activate $\mathrm{T}$ cells following activation with alloIgG-IC reflects a basic physiologic mechanism of immune regulation rather than a consequence of tumor-mediated suppression.

We next tested whether MoDC unresponsiveness to IC is present prior to differentiation or results from culture with GM-CSF. Inflammatory and patrolling monocytes were isolated from the peripheral blood of naive mice and were tested immediately for their ability to process alloIgG-IC. Even freshly isolated blood monocytes were unresponsive to alloIgG-IC, whereas BM monocytes significantly increased their expression of CD86 and MHC II and secreted detectable levels of IL-12 (Supplemental Figure 3A). In addition, mature $\mathrm{DC}\left(\mathrm{FSC}^{\mathrm{lo}} / \mathrm{SSC}^{\mathrm{lo}} / \mathrm{CD} 11 \mathrm{c}^{+} / \mathrm{MHC} \mathrm{II}^{+} / \mathrm{B} 220^{\text {neg }}\right)$ in the circulation also failed to upregulate activation markers following exposure to IC (Supplemental Figure 3B). Similar patterns of activation were also observed with MoDC obtained from B cell-deficient mice (Supplemental Figure 3B), excluding the 
A Untreated allolgG-IC Orthovanadate \begin{tabular}{c}
$\begin{array}{c}\text { Orthovanadate } \\
+ \text { allolgG-IC }\end{array}$ \\
\hline
\end{tabular}
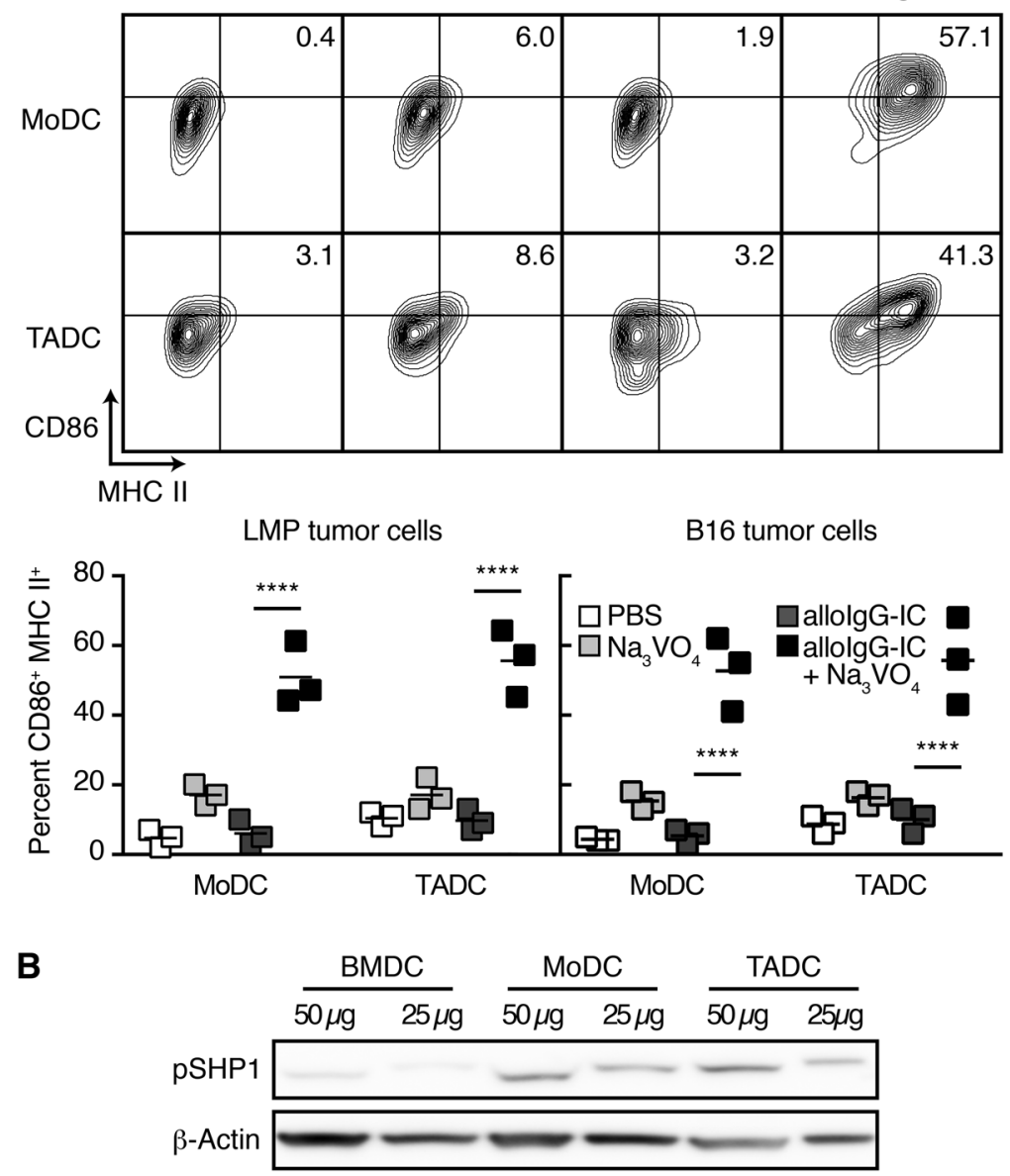

C

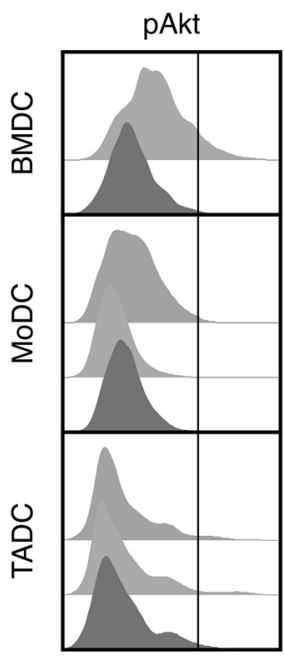

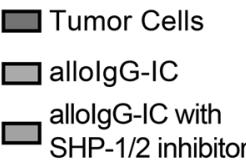

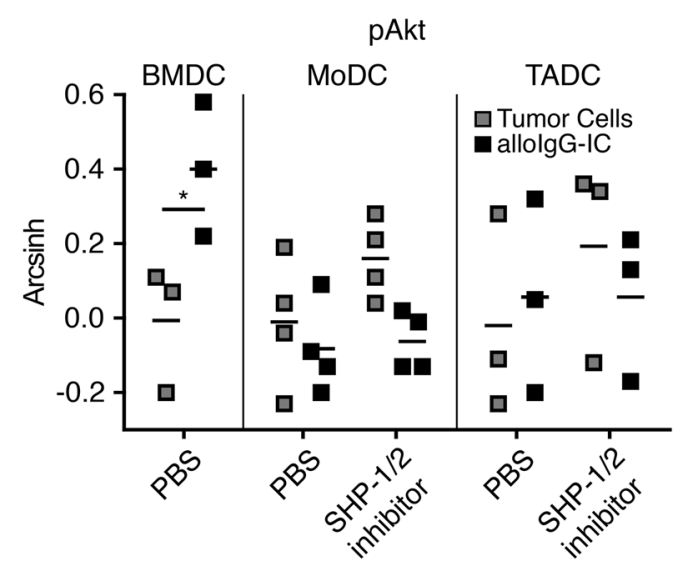

Figure 5. Broad blockade of protein tyrosine phosphatases enables activation of TADC and MoDC by allolgG-IC. (A) Flow cytometric analysis of MHC II and CD86 expression on blood monocyte-derived DC (MoDC) and tumor-associated DC (TADC) treated with orthovanadate (Na3VO4) following overnight incubation with allogeneic C57BL/6 IgG-LMP tumor cell immune complexes (allolgG-IC). Graphs show the percentages of DC expressing both MHC II and CD86. Statistical significance was determined by 2-way ANOVA with Sidak's multiple comparisons test. (B) Western blot analysis of phosphorylated SHP-1 (pSHP-1) in BM-derived DC (BMDC), MoDC, and TADC. Shown is 1 representative experiment of 4 performed. (C) Histograms show pAkt levels in DC after 10 minutes of incubation with LMP tumor cells or allogeneic C57BL/6 IgG-LMP tumor cell immune complexes (allolgG-IC) and with or without SHP-1/2 inhibitor. Dot plots show arcsinh ratios of pAkt in DC incubated for 10 minutes with allolgG-IC over baseline levels obtained from tumor cell-stimulated DC. These data also appear in Figure $6 \mathrm{~F}$ as a reference for comparison. Statistical significance for BMDC was assessed using Student's $t$ test with Welch's correction. Statistical significance for MoDC and TADC was determined by 2-way ANOVA with Tukey's multiple comparisons test. Graphs show the mean levels obtained in 1 representative experiment of 3 independently repeated experiments. ${ }^{*} P<0.05,{ }^{* * *} P<0.0001$.

possibility that Fc $\gamma$ Rs were merely already occupied on these cells. Moreover, similar results were obtained with monocytes and DC from mice lacking the inhibitory receptor Fc $\gamma$ RIIb (Supplemental Figure 3B), ruling out the possibility that the expression of the inhibitory FcR alone is responsible for abrogating signals through activating receptors. We also isolated hematopoietic stem cells $\left(\mathrm{Lin}^{\text {neg }} / \mathrm{c}-\mathrm{Kit}^{+} / \mathrm{Sca} 1^{+}\right)$from the circulation of G-CSF-treated mice and cultured them in GM-CSF to obtain DC. Similar to blood-derived monocytes and MoDC, these cells were unresponsive to alloIgG-IC (Supplemental Figure 3B).

Phosphorylation of Syk following alloIgG-IC uptake is inhibited in TADC and MoDC. To understand why DC obtained from the circulation or from tumors fail to respond to alloIgG-IC stimulation, we investigated the ability of these DC to phosphorylate key proteins downstream of the Fc $\gamma R$. Initially, we assessed the levels of phosphorylation of the MAPKs, which are known to play a requisite role in transmitting signals through the Fc $\gamma$ R. Fresh TADC and spleen DC, as well as cultured BMDC and MoDC, were activated with alloIgG-IC for 10 minutes, and the levels of the MAPK family members were quantified by flow cytometry using an established protocol (31). Marked increases in phosphorylated MAPK p38, ERK1/2, and JNK were observed in BMDC and, to a lesser extent, in splenic DC following incubation with alloIgG-IC (Figure 4A and Supplemental Figure 4A). In contrast, MoDC exhibited only low levels of MAPK phosphorylation (Figure 4A).

We next analyzed the phosphorylation levels of Akt, which reflect activation of the PI3K pathway and provide an important signal for DC survival and activation. DC subsets were isolated and activated with 
A

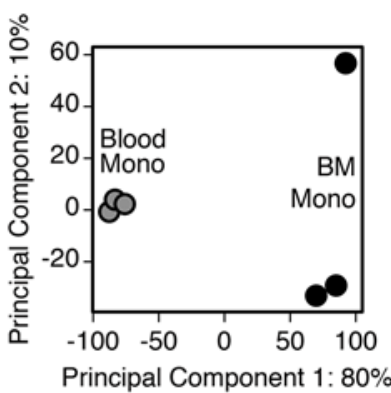

E BMDC MoDC TADC

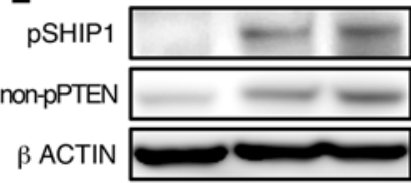

$\mathbf{F}$

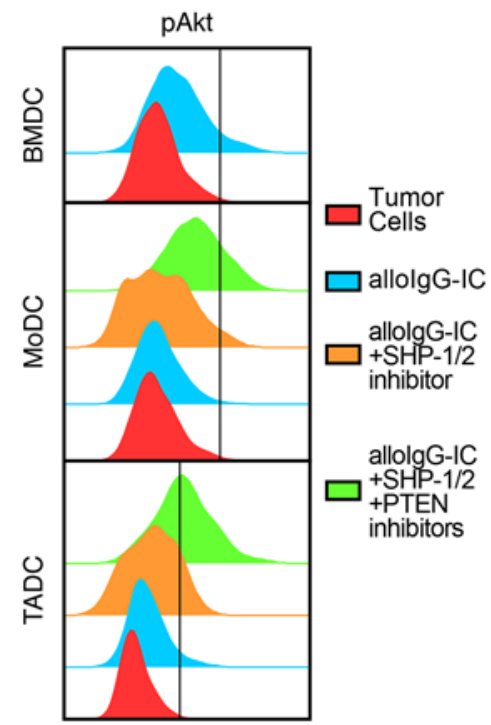

G MoDC
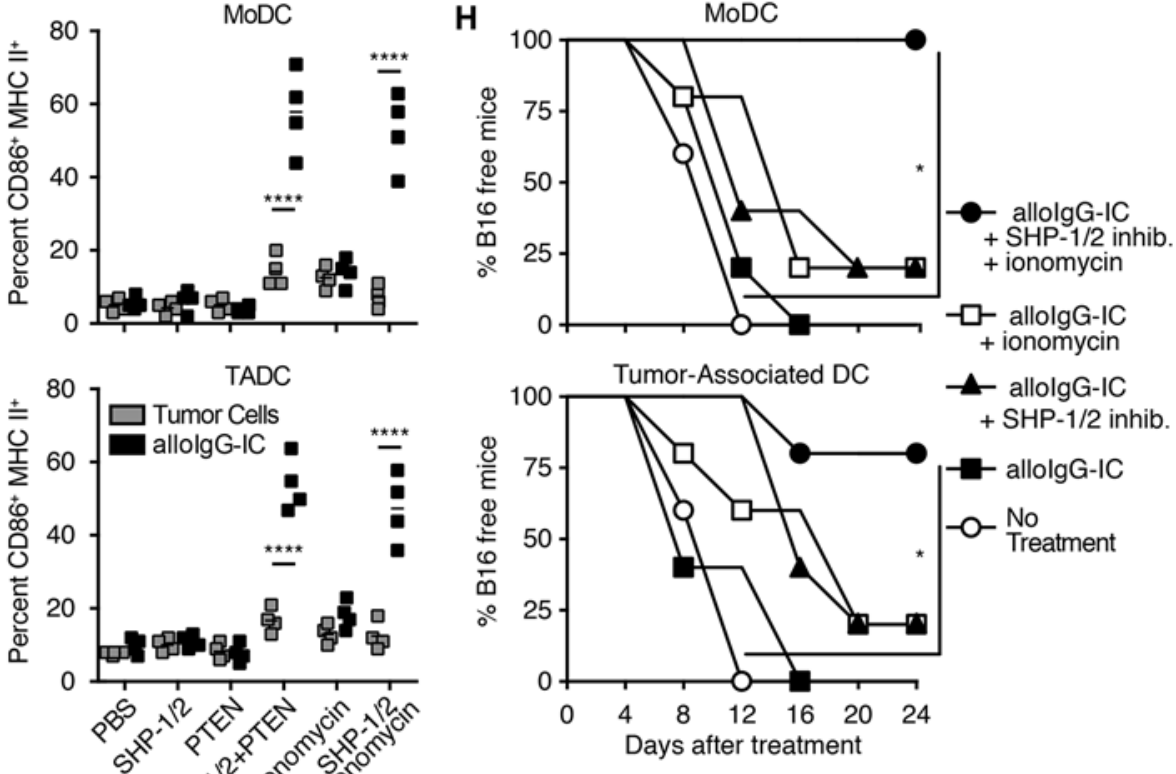

C Akt/PI3K Pathway Genes Canonical

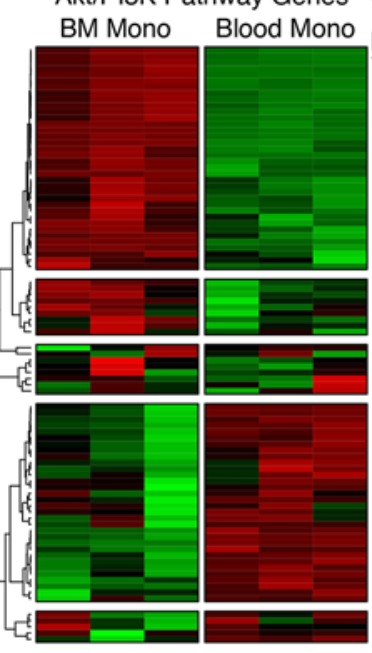

Pathways

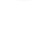

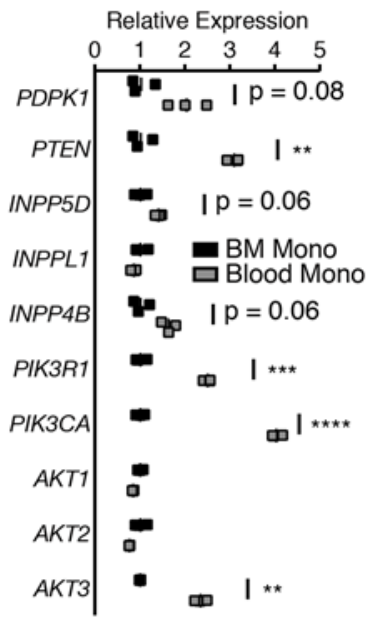

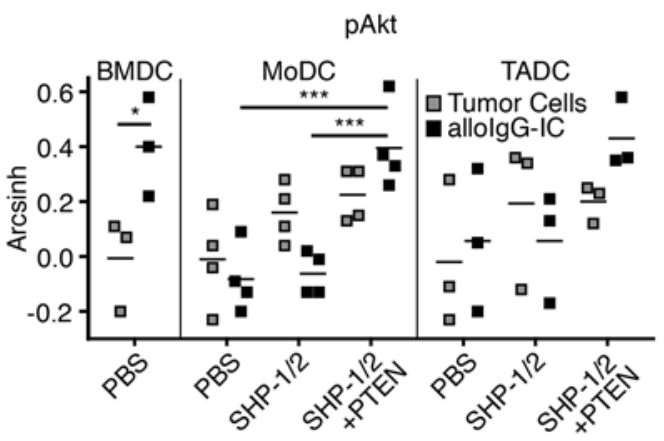


Figure 6. Simultaneous SHP-1 and PTEN blockade enables activation of TADC and MoDC. Gene expression in blood and BM monocytes from the ImmGen data sets analyzed by (A) principal component analysis, hierarchal clustering of (B) genome-wide data and (C) genes in the PI3K/Akt pathway, and (D) the most significantly altered genes closely involved in regulating Akt phosphorylation. Adjusted $P$ values and statistical significance were determined using the Holm-Sidak method with $\alpha=0.05$. (E) Western blots of phosphorylated SHIP-1 and nonphosphorylated PTEN in BM monocyte-derived DC (BMDC), blood monocyte-derived DC (MoDC), and tumor-associated DC (TADC). (F) Phosphorylated Akt levels in DC incubated with C57BL/6 allolgG-LMP tumor cell IC (allolgG-IC) over levels in tumor cell-stimulated DC in the presence of SHP-1/2 and PTEN inhibitors. Dot plots show arcsinh ratios of pAkt in DC incubated for 10 minutes with allolgG-IC over baseline levels obtained from tumor cell-stimulated DC. Part of these data also appear in Figure 5 C and are here as a reference for comparison. Statistical significance for BMDC was assessed using Student's $t$ test with Welch's correction. Statistical significance for MoDC and TADC was determined by 2-way ANOVA with Tukey's multiple comparisons test. (C) Flow cytometric analysis of MHC II and CD86 expression on DC subsets following overnight incubation with 129/Sv allogeneic IgG-B16 tumor cell immune complexes (allolgG-IC) and with or without specific phosphatase inhibitors. Graphs show the percentages of DC expressing both MHC II and CD86. Statistical significance was determined by 2-way ANOVA with Sidak's multiple comparisons test. (H) The rates of tumor recurrence in tumor-resected mice injected with MoDC or TADC preactivated with 129/Sv allogeneic IgG-B16 tumor cell immune complexes (allolgG-IC) and with or without phosphatase inhibitors. Statistical significance was determined by the log-rank Mantel-Cox test using Bonferroni-adjusted $P$ values. (I) Confocal and (J) flow cytometric analysis of uptake by MoDC from Fc $\gamma \mathrm{RIIb}^{-1-}$ mice after overnight incubation with CFSE-labeled B16 tumor cells in immune complexes with allogeneic 129/Sv IgC (allolgG-IC) and with or without PTEN inhibitor. Original magnification, $\times 40 .{ }^{*} P<0.05,{ }^{* *} P<0.01,{ }^{* * *} P<0.001,{ }^{* * *} P<0.0001$.

alloIgG-IC for 5 minutes before cell lysates were prepared for quantification using the PathScan Akt Signaling Antibody Array. Only BMDC exhibited strong phosphorylation of Akt, while spleen DC exhibited lower levels of the phosphorylated form (Figure 4B and Supplemental Figure 4B). Conversely, MoDC and TADC failed to significantly phosphorylate Akt following their incubation with alloIgG-IC (Figure 4B). We next sought to identify the point at which FcyR signal transduction is blocked in TADC and MoDC. Since phosphorylation of both MAPK and Akt is mediated by Syk, we investigated the phosphorylation of Syk in response to alloIgG-IC. Significant levels of phosphorylated Syk were found in BMDC within 1 minute of incubation with alloIgG-IC. In contrast, TADC and MoDC exhibited substantially delayed and marginal Syk phosphorylation (Figure 4C), demonstrating that Fc $\gamma \mathrm{R}$ signaling is differentially regulated very early in the signaling cascade.

Broad blockade of protein tyrosine phosphatases in TADC and MoDC enables activation by alloIgG-IC. Since tyrosine phosphatases are the most extensively documented regulators of $\mathrm{F} c \gamma \mathrm{R}$ signaling, and in particular of Syk phosphorylation $(23,32)$, we treated TADC and MoDC with sodium orthovanadate, a broad protein tyrosine phosphatase inhibitor, before incubation with alloIgG-IC. Strikingly, phosphatase inhibition enabled activation of all DC subsets, as evident by upregulation of CD86 and MHC II (Figure 5A). The phosphatase SHP-1 is known to inhibit Syk phosphorylation in other contexts (33). Indeed, levels of the active, phosphorylated form of SHP-1 were markedly lower in BMDC compared with TADC and MoDC (Figure 5B). The thoroughly characterized molecule coupled with SHP-1 that regulates Fc $\gamma \mathrm{R}$ signaling is the inhibitory receptor Fc $\gamma \mathrm{RIIb}$ (25). However, incubation of MoDC from mice lacking Fc $\gamma$ RIIb with IC did not result in significant DC activation, similar to MoDC from WT mice (Supplemental Figure 3B). Therefore, we next tested if specific inhibition of SHP-1 was sufficient to enable activation of otherwise unresponsive DC. Although blocking SHP-1 enabled phosphorylation of Syk and its downstream MAPK protein Erk1/2 (data not shown), neither MoDC nor TADC could internalize alloIgG-IC following SHP-1 inhibition. Additionally, even with high doses of SHP-1 inhibitor, these cells did not exhibit Akt phosphorylation following stimulation with alloIgG-IC (Figure 5C).

Simultaneous blockade of SHP-1 and phosphatases regulating Akt enables tumor and MoDC activation to facilitate tumor rejection. We next investigated whether additional tyrosine phosphatases might prevent the activation of MoDC and TADC by alloIgG-IC. Initially, we analyzed gene expression data from the Immunological Genome Project (34), comparing mRNA expression levels in blood and BM monocytes. Principal component analysis clearly distinguished BM monocytes from blood monocytes (Figure 6A). Genes with statistically significant $(P<0.05)$ differences in expression between these populations were analyzed using Ingenuity Pathway Analysis software. The top canonical pathway in BM monocytes was cell cycle regulation, which was not surprising given that the $\mathrm{BM}$ is the site of origin of monocytes. Interestingly, the top canonical pathway in MoDC was PI3K signaling (Figure 6B). Further analysis of genes involved in the PI3K pathway demonstrated dramatic alteration in gene expression related to phosphatase and tensin homolog (PTEN) and Fc $\gamma$ R signaling (Figure 6C). Of interest, blood monocytes express significantly higher levels of genes regulating the PI3K/Akt pathway (Figure 6D). To determine whether these changes are also present in MoDC, TADC, and BMDC, we compared the levels of phosphorylated Src homology 2 domain-containing inositol polyphosphate phosphatase 1 (SHIP-1) and nonphosphorylated PTEN, which 
are the active forms of these phosphatases, in these cells. In fact, lower levels of PTEN and dramatically reduced levels of SHIP-1 were observed in BMDC, suggesting that the PI3K/Akt pathway is differentially regulated in MoDC and TADC (Figure 6E).

To assess whether inhibitors of the PI3K/Akt pathway inhibitory phosphatases could enable DC activation following incubation with IgG-IC, we used the well-characterized PTEN inhibitor, bisperoxovanadate, or the SHIP-1 inhibitor, $3 \alpha$-aminocholestane, to selectively block their activity. AlloIgG-IC-pulsed MoDC and TADC were treated with SHIP-1 or PTEN inhibitor alone or in combination with the SHP-1/2 inhibitor. Only the combination of the SHP-1/2 inhibitor with either the PTEN inhibitor or the SHIP-1 inhibitor induced striking Akt phosphorylation in MoDC and TADC following their incubation with alloIgG-IC (Figure 6F and Supplemental Figure 5A). Consistently, only when both SHP-1 and phosphatases regulating Akt were inhibited did MoDC and TADC internalize alloIgG-IC and upregulate costimulatory molecules (Figure 6G and Supplemental Figure 5B). As an alternative method to directly activate the PI3K/Akt pathway, we treated DC with the ionophore ionomycin to induce influx of calcium ions. Similar to blocking SHP-1 and phosphatases regulating Akt (e.g., PTEN and SHIP-1), induction of Akt signaling using the calcium ionophore ionomycin enabled MoDC and TADC to upregulate costimulatory molecules when treated with alloIgG-IC in the presence of SHP-1/2 inhibitor (Figure 6G). We next tested the capacity of these DC to protect mice from tumor challenge in vivo. MoDC and TADC were cultured overnight with alloIgG-IC alone or in combination with SHP-1/2 inhibitor and ionomycin and then s.c. injected naive mice. After 5 days, mice were challenged with B16 melanoma cells, and tumor development was monitored. Consistent with the results in culture, only mice injected with either MoDC or TADC that were loaded with alloIgG-IC in the presence of both the SHP-1/2 inhibitor and ionomycin could protect mice from tumor growth (Figure $6 \mathrm{H}$ ). Taken together, these results demonstrate that blocking SHP-1 in MoDC and TADC is not sufficient to enable their activation by IC and that Akt phosphorylation is an additional checkpoint for DC activation.

Given the known association of SHP-1 with the inhibitory Fc $\gamma$ RIIb, we hypothesized that, in the absence of FcrRIIb, PTEN inhibition alone would enable MoDC to respond to alloIgG-IC. Blood monocytes were isolated from mice lacking Fc $\gamma$ RIIb and cultured with GM-CSF to obtain DC, which were then activated with alloIgG-IC alone or in the presence of PTEN inhibitor. Consistent with our previous results, treatment of MoDC with alloIgG-IC did not result in significant activation and was comparable to that of MoDC from WT mice. In support of our hypothesis, however, blockade of PTEN in MoDC derived from FcyRIIb KO mice was sufficient to induce alloIgG-IC uptake and activation (Figure 6, I and J, and Supplemental Figure 5C).

Activation of human TADC and MoDC by alloIgG-IC is regulated similarly to mouse DC. To assess these pathways of DC activation in the human setting, BM-derived CD $34^{+}$cells, CD $14^{+}$, and $\mathrm{CD} 11 \mathrm{c}^{+} / \mathrm{MHC} \mathrm{II}^{+}$cells derived from the lungs of mesothelioma patients were cultured in medium containing GM-CSF to obtain DC. DC were then incubated overnight with fixed autologous tumor cells alone or in combination with syngeneic IgG or allogeneic IgG from 10 pooled donors. DC were washed, and autologous CD4 ${ }^{+} \mathrm{T}$ cells from the corresponding patient were added to the culture. In both patients, untreated BMDC, MoDC, and TADC did not result in significant $\mathrm{CD}^{+} \mathrm{T}$ cell proliferation (Figure 7A). Similarly, activation of BMDC, MoDC, and TADC with IgG-IC formed with autologous antibodies (self-IgG) induced only low CD4 ${ }^{+}$ $\mathrm{T}$ cell proliferation. As in our studies of murine BMDC, incubation of human BMDC with alloIgG-IC induced a 4- to 5-fold increase in $\mathrm{CD}^{+} \mathrm{T}$ cell proliferation compared with BMDC incubated with selfIgG-IC. In contrast, MoDC and TADC cultured under these conditions failed to stimulate $\mathrm{CD}^{+} \mathrm{T}$ cell proliferation (Figure 7A).

We next tested if the inability of MoDC to induce $\mathrm{T}$ cell proliferation results from inefficient IC internalization. To compare the capacity of each DC subset to take up IC, we labeled autologous tumor cells from mesothelioma patients with CFSE, incubated these cells with alloIgG, and incubated them overnight with either BMDC or MoDC. DC were then washed, and CFSE in these cells was measured by microscopy and flow cytometry. Again, consistent with our mouse results, MoDC internalized only marginal levels of autologous tumor cells coated with allogeneic IgG, whereas BMDC efficiently internalized these cells (Figure 7B).

Our murine studies demonstrated that the difference in activation between MoDC and BMDC was not unique to the cancer setting, but rather represented a generalizable principle of regulation. To assess the responsiveness of healthy human BMDC and MoDC to alloIgG-IC, BMDC and MoDC were generated 

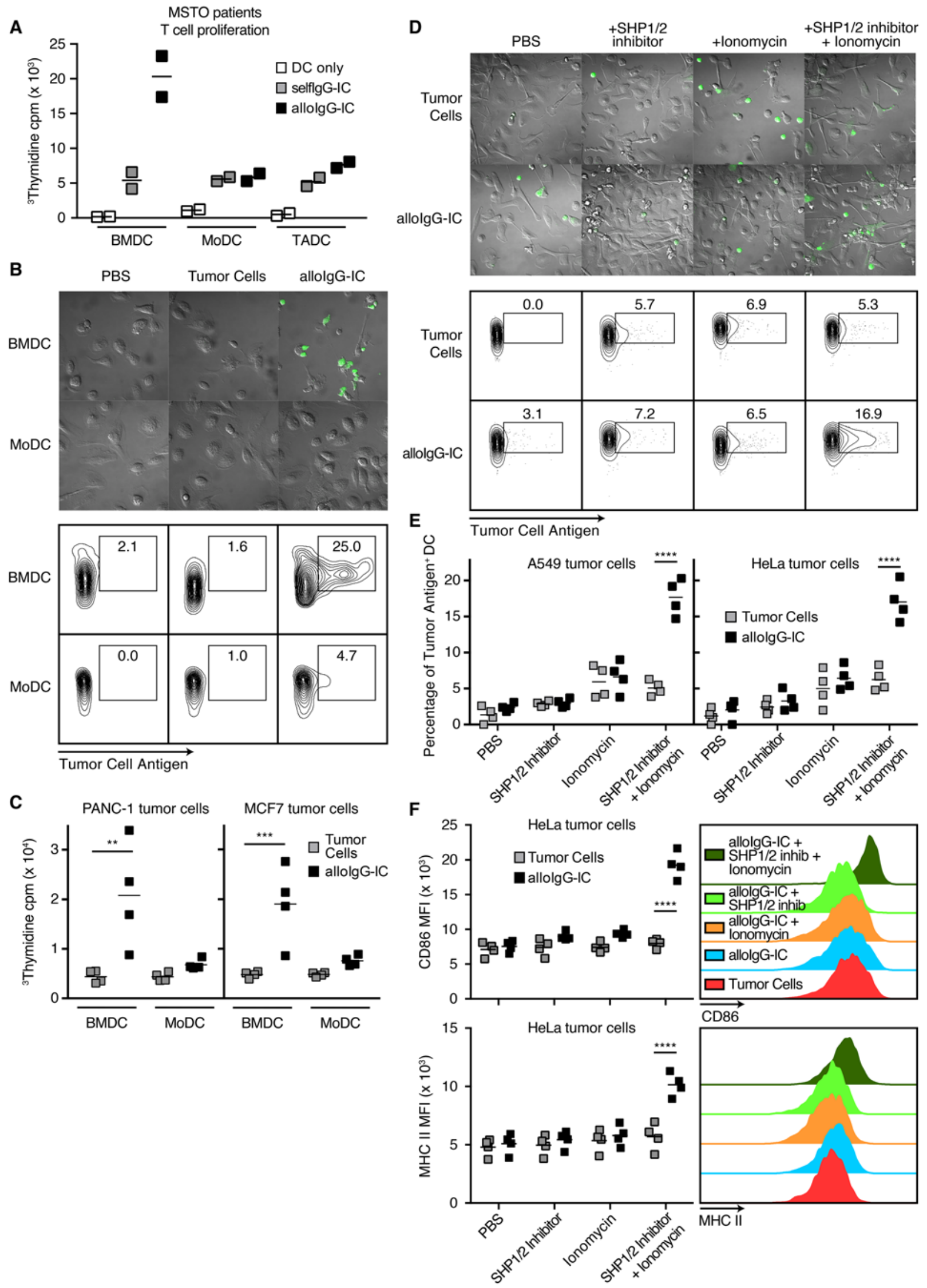
Figure 7. Activation of human MoDC by allolgG-IC is regulated by both SHP-1 and calcium signaling. (A) Proliferative response of CD4 ${ }^{+} T$ cells from mesothelioma (MSTO) patients after 6-day culture with autologous BM monocyte-derived DC (BMDC), blood monocyte-derived DC (MoDC), or tumor-associated DC (TADC) and with self-IgG- or allolgG-coated autologous tumor cells (self-IgG-IC or allolgG-IC, $n=2$ ). (B) Uptake by autologous DC of CFSE-labeled MSTO tumor cells (green) that were untreated or coated with allogeneic IgC (allolgG-IC, $1 \mu \mathrm{g}$ per $1 \times 10^{5}$ cells) $(n=2)$. Original magnification, $\times 40$. (C) Proliferative response of CD4+ $T$ cells from healthy donors after 6-day culture with autologous DC preactivated with tumor cells that had been incubated or not with intravenous Ig (IVIG, $n=5$ ). (D) Uptake by MoDC from healthy donors of CFSE-labeled A549 tumor cells (green) incubated with allogeneic IgC $\left(1 \mu \mathrm{g}\right.$ per $1 \times 10^{5}$ cells). Photomicrographs show 1 representative donor of 6 tested. Original magnification, $\times 40$. (E) The effect of phosphatase inhibitors on the uptake of CFSE-labeled IC by MoDC from healthy donors $(n=4)$. (F) Mean fluorescence intensity (MFI) of CD86 and MHC II on MoDC following overnight incubation with allolgG-IC in the presence or absence of phosphatase inhibitors $(n=4)$. Histograms show the mean levels obtained in 1 representative experiment of 4 independently repeated experiments. Statistical significance was determined by 2-way ANOVA with Sidak's multiple comparisons test. ${ }^{* *} P<0.01,{ }^{* *} P<0.001$, ${ }^{* * *} P<0.0001$.

from monocytes isolated from matched healthy donors, and their capacity to promote autologous $\mathrm{CD} 4^{+} \mathrm{T}$ cell proliferation following activation with alloIgG-IC was measured. Consistently, the proliferation rates of $\mathrm{CD}^{+} \mathrm{T}$ cells cultured with BMDC loaded with alloIgG-IC were significantly higher than those cultured with MoDC (Figure 7C). We next assessed the role of SHP-1 and PTEN in regulating the activation of human BMDC and MoDC. Because human DC myeloperoxidase inactivates PTEN small-molecule inhibitors (by adding $\mathrm{H}^{+}$to $\mathrm{VO}_{4}^{3-}$ oxyanion), we used ionomycin to activate the PI3K/Akt pathway. Consistent with our results in mice, only MoDC cultured with CFSE-labeled tumor cells in the presence of SHP-1/2 inhibitors and ionomycin internalized tumor antigens (Figure 7, D and E). In addition, these cells elevated their expression of CD86 and HLA-DR, indicating that blocking these phosphatases enabled their activation by IC (Figure 7F). Similar to MoDC, classical and plasmacytoid DC isolated from human blood were not responsive to IC. However, incubation with ionomycin and SHP-1/2 inhibitor at levels equivalent to those used with MoDC did not enable their activation by IC (data not shown). It may be that different concentrations of ionomycin and SHP-1/2 inhibitor are required or that additional phosphatases regulating Fc $\gamma$ Rs are upregulated in these cells. Overall, these results show that human DC subsets respond to alloIgG-IC in a similar manner to their mouse DC counterparts.

\section{Discussion}

Attempts to employ DC loaded with IC to treat established tumors, rather than as prophylactic tumor vaccines, have achieved only marginal success in clinical trials (reviewed in ref. 20). These findings are surprising given the extensive work showing that uptake of IC by DC leads to efficient antigen presentation and strong $\mathrm{T}$ cell activation in vitro and in vivo $(28,35-38$, and reviewed in refs. 20,39$)$. In a recent study, we found that, in contrast to BMDC, which have been used for most mouse DC vaccination studies, TADC require additional stimulatory signals in order to process and present antigens derived from IC to $\mathrm{T}$ cells (22). However, the molecular mechanism that governs this phenomenon remained elusive.

Our results demonstrate in both humans and mice that the unresponsiveness of TADC to IC is not due to the suppressive nature of the tumor microenvironment; rather, it is a consequence of normal monocyte maturation. Activation of two major Fc $\gamma \mathrm{R}$ signaling pathways, specifically MAPK and $\mathrm{PI} 3 \mathrm{~K} / \mathrm{Akt}$, is critical for MoDC to respond to IC. In contrast to MoDC and TADC, which require inhibition of phosphatases for IC-dependent activation, BMDC exhibit lower basal phosphatase activity and are consequently capable of IC-driven activation and induction of an antitumor T cell response without phosphatase inhibition.

Examination of signaling pathways in MoDC, TADC, and BMDC demonstrated that, while rapid Syk phosphorylation following ligation of Fc $\gamma$ Rs with IC is induced in BMDC, it is not induced in MoDC or TADC. Consistent with previous reports (40), this early Syk phosphorylation yields dramatic activation of downstream proteins in both the MAPK (p38, pJNK, pERK) and PI3K/Akt (pAkt) pathways in BMDC. Although the phosphatase SHP-1 is known to regulate DC activation as well as Syk phosphorylation (41), inhibition of SHP-1 alone was not sufficient to enable MoDC or TADC to respond to IC in MoDC and TADC. SHP-1 has previously been implicated in regulating DC activation. The motheaten mouse, which bears a deletion mutation of SHP-1 alone in hematopoietic cells, develops severe spontaneous autoimmunity (42). Moreover, Abram et al. have demonstrated recently that deletion of SHP-1 in DC is sufficient to induce autoimmunity in mice (43). Similar observations were made by Kaneko et al., who showed deposition of autoantibodies in the lungs and kidneys of mice bearing DC-specific SHP-1 ablation starting after 36 weeks (44). 
Our data show that SHP-1, acting via Fc $\gamma$ RIIb, is the major early signaling checkpoint restricting MoDC activation. Dhodapkar et al. previously observed that human MoDC do not respond to IC but attributed this to augmentation of the inhibitory Fc $\gamma$ RIIb and to unknown factors present in serum (45). Our data support this idea in part. However, we found that both MoDC and TADC have markedly elevated levels of SHP-1 and phosphatases that regulate Akt, which prevents their signaling through $\mathrm{F}_{\mathrm{c}} \gamma \mathrm{R}$ and thus antigen uptake and presentation. Prior studies have indicated that FcyRIIb deficiency acts to amplify spontaneous autoimmunity, yet it is not sufficient to initiate it (46-49). Given this background, it is therefore not clear why Fc $\gamma$ RIIb-deficient mice do not exhibit spontaneous autoimmune disease, similar to the motheaten mouse. Interestingly, we found that Fc $\gamma$ RIIb-deficient mice express substantially lower levels of all activating Fc $\gamma$ Rs (i.e., Fc $\gamma$ RI, Fc $\gamma$ RIII, and Fc $\gamma$ RIV) compared with the levels present in WT mice (data not shown), and phosphatase regulation of PI3K/Akt signaling provides an additional checkpoint even in this setting.

Activation of Syk alone in myeloid cells is considered sufficient to activate the PI3K/Akt pathway (reviewed in refs. 41, 50). In contrast, we found that the PI3K pathway is suppressed in MoDC and TADC and that inhibition of SHP-1 alone is not sufficient to promote Akt signaling and DC activation by IC, but rather requires inhibition of PTEN or SHIP-1. Most prior studies have shown the necessity of PI3K/ Akt for metabolic adaptation of DC to the tumor microenvironment (51) and for induction of successful DC-based immunity (52). In accordance with these findings, mice bearing DC-specific deletion in PTEN, SHIP-1, or inositol polyphosphate-4-phosphatase exhibited increased PI3K/Akt signaling and were shown to have increased DC activity and unattenuated inflammation in their lungs (53-57). Consistently, blocking $\mathrm{PI} 3 \mathrm{~K} / \mathrm{Akt}$ in DC using rapamycin was shown to almost completely abrogate their capacity to secrete IFN- $\gamma$ and to present $\mathrm{T}$ cell antigens $(58,59)$.

Importantly, studies suggesting that Syk phosphorylation is sufficient for PI3K/Akt activation have relied on genetic mouse models in which complete SHP-1 loss was induced. In contrast, we used pharmacological inhibitors, which may provide only partial inhibition. Therefore, it may be that with complete SHP-1 deletion Syk activation can overcome the PTEN/SHIP-1 checkpoint that restricts Akt activation. Nevertheless, combined inhibition of both SHP-1 and PTEN or SHIP-1 rendered MoDC and TADC responsive to activation by IC. These results demonstrate that two checkpoints exist for IC-driven DC activation in peripheral MoDC.

Finally, while the positive role of antibodies in promoting immunity during bacterial infection is well established, their role during tumor progression is still controversial. A number of studies have described how engagement of Fc $\gamma$ Rs by IC induces an immunosuppressive phenotype in various tumor-infiltrating cells, including DC (60-62), macrophages, mast cells, and B cells (63-65, and reviewed in ref. 10). However, these findings remain surprising given the extensive evidence that uptake of IC by DC leads to antigen presentation and elicits a strong $\mathrm{T}$ cell immune response (28, 29, 35-38, and reviewed in ref. 20, 39). The present work provides insight into this question by identifying the intrinsic checkpoints that regulate the activation of TADC and MoDC by IC, demonstrating that modulation of these checkpoints can enable the induction of potent antitumor immunity. Thus, in the absence of sufficient DC stimuli, antibodies may contribute to immune suppression by clearing antigens that would otherwise be available for immunosurveillance by armed effector cells. This may also explain why administration of intravenous Ig can induce DC-mediated tolerance, as low antigen presentation in the absence of a stimulatory context is most likely to induce regulatory or anergic $\mathrm{T}$ cells $(66,67)$. Conversely, in the presence of appropriate stimuli (e.g., TLR agonists) DC can process IC and present their processed antigens to T cells. These studies thus resolve outstanding controversies in the field of DC-mediated antitumor immunity, paving the way for effective clinical manipulation of these pathways.

\section{Methods}

Mice. 129S1/SvlmJ mice, C57BL/6 WT mice, B cell knockout mice (B6.129S2-Ighm $\left.{ }^{\text {tm }} 1 \mathrm{Cgn} / \mathrm{J}\right)$, CD45.1 mice (B6.SJL-Ptprca Pepcb/BoyJ), and $\mathrm{Fc}_{\mathrm{R}} \mathrm{RIIb}^{-/-}\left(B 6.129 S 4-\mathrm{F}_{\left.\mathrm{c} g r 2 b^{t m I T t K}\right)}\right.$ mice were purchased from The Jackson Laboratory and bred onsite. 12- to 16-week-old mice were placed randomly into groups before assigning treatment conditions. All mice were maintained in an American Association for the Accreditation of Laboratory Animal Care-accredited animal facility.

Cell lines. The mouse melanoma cell line B16F10 and the human cell lines HeLa, MCF-7, PANC-1, and A549 were purchased from ATCC. LMP pancreatic adenocarcinoma cells were isolated from $\mathrm{Kras}^{G 12 \mathrm{D} /+}$; 
LSL-Trp53 ${ }^{R 172 H /+} ;$ Pdx-1-Cre mice as described previously (68). Cells were cultured in DMEM (Gibco) supplemented with $10 \%$ heat-inactivated FCS, $2 \mathrm{mM}$ L-glutamine, $100 \mathrm{U} / \mathrm{ml}$ penicillin, and $100 \mu \mathrm{g} / \mathrm{ml}$ streptomycin (Gibco) under standard conditions. Prior to their use, cells were tested for endotoxins using the LAL Chromogenic Endotoxin Quantitation Kit (Pierce) and for mycoplasma using PlasmoTest (InvivoGen), according to manufacturer's instructions.

Preparation of mouse DC subsets. For BMDC, BM mononuclear cells were negatively enriched using a murine monocyte enrichment kit (Stem Cell Technologies), and $\mathrm{FSC}^{\mathrm{lo}} / \mathrm{SSC}^{\mathrm{lo}} / \mathrm{Ly} 6 \mathrm{C}^{+} / \mathrm{CD} 115^{+}$cells were sorted with a FACS Aria II (BD Biosciences). Monocytes were cultured for 4 to 5 days in the presence of $50 \mathrm{ng} / \mathrm{ml}$ GM-CSF (PeproTech) to generate DC.

For TADC, tumors were digested in HBSS (Gibco) containing $4 \mathrm{mg} / \mathrm{ml}$ collagenase IV and $0.01 \mathrm{mg} /$ $\mathrm{ml}$ DNase I (Sigma-Aldrich). Cells were applied to a Ficoll gradient and magnetically enriched using $\mathrm{CD}_{11 \mathrm{~b}^{+}}$selection kits (Stemcell Technologies) and $\mathrm{FSC}^{\mathrm{lo}} / \mathrm{SSC}^{\mathrm{lo}} / \mathrm{Ly} 6 \mathrm{C}^{\text {neg }} / \mathrm{CD} 11 \mathrm{c}^{+} / \mathrm{MHC}^{\mathrm{hi}}$ cells were sorted by FACS. For MoDC, peripheral blood cells were applied to a Ficoll gradient and magnetically enriched using $\mathrm{CD}_{11 b^{+}}$selection kits (Stemcell Technologies). Patrolling monocytes were sorted as $\mathrm{FSC}^{\mathrm{lo}} /$ $\mathrm{SSC}^{\mathrm{lo}} / \mathrm{Ly} 6 \mathrm{C}^{\mathrm{neg}} / \mathrm{CD} 115^{+}$cells and inflammatory monocytes were sorted as $\mathrm{FSC}^{\mathrm{lo}} / \mathrm{SSC}^{\mathrm{lo}} / \mathrm{Ly} 6 \mathrm{C}^{+} / \mathrm{CD} 115^{+}$ by FACS. For spleen DC, spleens were mashed through 70-micron mesh filters, and DC were negatively enriched using the Pan-DC negative selection kit (Miltenyi). Subsequently, FSC ${ }^{\mathrm{lo}} / \mathrm{SSC}^{\mathrm{lo}} / \mathrm{CD} 1 \mathrm{C}^{+} / \mathrm{MHC}$ II $^{\text {hi }}$ cells were sorted with a FACS Aria II and used immediately. All assays of in vitro activation of mouse DC were independently repeated at least 5 times in technical duplicates.

Preparation of human $B M$ and $M o D C$. For MoDC, peripheral blood of healthy individuals was applied to a Ficoll gradient (BD). $\mathrm{CD}_{14}^{+}$cells were isolated from mononuclear cells using magnetic beads (Miltenyi). Cells were then cultured for 6 days in RPMI containing 10\% pooled human serum, $50 \mathrm{ng} / \mathrm{ml}$ GM-CSF (PeproTech), and $20 \mathrm{ng} / \mathrm{ml}$ IL-4 (PeproTech). For some experiments, BM cells and peripheral blood from the same donor were purchased from AllCells. The cells were applied to a Ficoll gradient (BD), and CD34 cells were isolated from mononuclear cells using magnetic beads (Miltenyi). Cells were then cultured for 6 days in RPMI containing 10\% pooled human serum, $50 \mathrm{ng} / \mathrm{ml} \mathrm{GM-CSF}, 20 \mathrm{ng} / \mathrm{ml} \mathrm{IL-4}$, and $20 \mathrm{ng} / \mathrm{ml}$ TNF (all from PeproTech).

Preparation and in vitro studies of tumor cells, TADC, autologous $T$ cells, and IgG from patients with cancer. Tumor cells, TADC, peripheral blood T cells, and IgG were obtained from 2 patients undergoing resection surgery for stage I lung carcinoma. Tumors were enzymatically digested with $0.1 \mathrm{mg} / \mathrm{ml}$ DNase I and 5 $\mathrm{mg} / \mathrm{ml}$ collagenase IV (Sigma-Aldrich) in HBSS for 30 minutes. Tumor cells were enriched by sorting CD45- cells, fixed in 2\% paraformaldehyde for 10 minutes, washed extensively in PBS, and coated for 30 minutes with autologous IgG or pooled allogeneic IgG obtained from healthy blood donors. To obtain TADC, $\mathrm{FSC}^{\mathrm{lo}} / \mathrm{SSC}^{\mathrm{lo}} / \mathrm{CD} 11 \mathrm{c}^{+} / \mathrm{MHC} \mathrm{II}^{\mathrm{hi}}$ cells were sorted and maintained for 1 hour in $10 \%$ FCS IMDM at $37^{\circ} \mathrm{C}$. For FACS and confocal studies, tumor DC were incubated overnight with autologous tumor cells coated with self-IgG or alloIgG. In separate experiments, $10-\mathrm{cm}$ long rib bones and $10 \mathrm{ml}$ blood were obtained from 2 patients undergoing resection surgery for malignant pleural mesothelioma. To generate BMDC, bones were flushed with PBS and mononuclear cells were separated on Ficoll gradients. CD34 cells were then enriched using magnetic beads (Miltenyi) and cultured for 9 days in IMDM (Gibco) supplemented with 10\% FCS, $50 \mathrm{ng} / \mathrm{ml}$ human GM-CSF, $20 \mathrm{ng} / \mathrm{ml}$ human IL-4, and $20 \mathrm{ng} / \mathrm{ml} \mathrm{TNF}$ (PeproTech). To obtain autologous tumor cells, tumors were enzymatically digested with $0.1 \mathrm{mg} / \mathrm{ml}$ DNase I and $5 \mathrm{mg} / \mathrm{ml}$ collagenase IV (Sigma-Aldrich) in HBSS for 30 minutes. Tumor cells were enriched by sorting CD45- cells, fixed in 2\% paraformaldehyde for 10 minutes, washed extensively in PBS, and coated for 30 minutes with autologous or pooled allogeneic IgG. Autologous $\mathrm{CD} 4^{+} \mathrm{T}$ cells were enriched from peripheral blood mononuclear cells on magnetic beads (Miltenyi), and IgG was isolated from each patient's plasma using protein A columns (GE Healthcare). For T cell proliferation assays, $2 \times 10^{4} \mathrm{DC}$ were incubated overnight with antibody-coated tumor cells as above, washed, and cocultured with $2 \times 10^{5}$ autologous CD $4^{+}$-enriched T cells. After 6 days, cells were pulsed with ${ }^{3} \mathrm{H}$-thymidine $(1 \mu \mathrm{Ci} /$ well $)$ and cultured for an additional 18 hours before being harvested in a Harvester 400 (Tomtec). Radioactivity was measured by a 1450 MicroBeta counter (LKB Wallac). T cell proliferation was assayed in 6 technical replicates per sample.

Phospho-AKT, phospho-SHP-1, phospho-SHIP-1, and nonphospho-PTEN blots. For phosphorylated AKT using the signaling array, $2 \times 10^{6} \mathrm{DC}$ were incubated with LMP-IgG IC for 1 minute and washed twice with PBS, and samples were further processed following the manufacturer's instructions (Cell Signaling Technology, PathScan Akt Signaling Antibody Array). 
For phosphorylated proteins, $2 \times 10^{6} \mathrm{DC}$ were activated with LMP-IgG IC for 1 minute, washed twice with PBS, and immediately lysed in RIPA buffer (Sigma-Aldrich) containing proteinases and phosphatases inhibitors (Sigma-Aldrich). Protein concentration was determined by the BCA protein assay kit (Life Technologies), and aliquots were mixed with loading buffer (Cell Signaling Technology) and run for 2 hours in a Novex PAGE Bis-Tris gel system (Life Technologies). Bands were transferred to nitrocellulose membrane and incubated overnight with rabbit mAb anti-mouse phospho-SHP-1 (clone D11G5, dilution 1:1,000), rabbit polyclonal anti-phospho-SHIP-1 (Tyr1020) (dilution 1:1,000), or rabbit polyclonal nonphospho-PTEN (Ser380/Thr382/Thr383). All antibodies were purchased from Cell Signaling Technology. The membranes were washed, incubated for 45 minutes with goat anti-rabbit antibodies conjugated to HRP (Cell Signaling), developed with SuperSignal West Femto Substrate (Life Technologies), and imaged.

Flow cytometry. For mouse cell surface staining, monoclonal antibodies conjugated to FITC, PE, PE-Cy7, PE-Cy5.5, APC-Cy7, eFluor 650, or Pacific Blue and antibodies specific for the following antigens were used: CD11b (M1/70), F4/80 (BM8), and B220 (RA3-6B2) from BioLegend and CD115 (AFS98), CD80 (16-10A1), I-Ab (AF6-120.1), CD40 (1C10), Ly6C (HK1.4), and CD86 (GL1) from eBioscience. All in vivo experiments to characterize tumor-infiltrating leukocytes were independently repeated at least 3 times with 3 to 5 mice per group. For protein phosphorylation-specific flow cytometry, cells were activated for 5,15 , or 30 minutes with or without IC and fixed for 15 minutes with $1.8 \%$ paraformaldehyde. Cells were washed twice with PBS containing $2 \%$ FCS and incubated with $95 \%$ methanol at $4^{\circ} \mathrm{C}$ for 20 minutes. Fluorochrome-conjugated antibodies against phospho-p38 (Thr180/Tyr182), phospho-JNK (Ser63), phospho-ERK1/2 (p44) (pT202/pY204), and phospho-Akt (pY473) were purchased from Cell Signaling Technologies. DC protein phosphorylation experiments were repeated 2-4 times. For human cell surface staining, the following conjugated monoclonal antibodies were used: APC-Cy7 CD86 (clone IT2.2) and Cy7 CD11c (clone Bu15) from BioLegend and V500 HLA-DR (clone G46-6) from BD Biosciences.

Cytokine measurements. Cells were seeded at $1 \times 10^{6}$ cells $/ \mathrm{ml}$ and cultured for 12 hours with or without tumor IC or LPS (Sigma-Aldrich). TNF, IFN- $\gamma$, and IL-12 (p40/p70) in the supernatants were measured by ELISA, according to manufacturer's instructions (R\&D Systems). Cytokine secretion was measured in biological triplicates in 4 independent experiments.

IgG purification and measurement. Mouse allogeneic antibodies were obtained from pooled 5-ml 20- to 24-week-old mouse serum by liquid chromatography on AKTA Explorer/100Air (GE Healthcare). Total mouse IgG was purified using protein-G (GE Healthcare). The levels of purified IgG were measured with specific ELISA kits (Bethyl) according to manufacturer's instructions. The capacity of purified antibodies to bind tumor cells was tested by flow cytometry prior to their use in vivo. $1 \mu \mathrm{g}$ IgG per $1 \times 10^{5}$ allogeneic tumor cells bound at least 5 times higher compared with isotype control antibodies. Serum levels of antibodies were measured in biological triplicates in 4 independent experiments.

Preparation of antibody-tumor cell IC. When obtained from surgical resections, tumor cells were initially isolated after enzymatic digestion and sorted as $\mathrm{FSC}^{\text {hi }} / \mathrm{CD}^{4} 5^{\text {neg }}$ cells prior to their fixation and staining. For tumor-antibody complexes, tumor cells were fixed in $2 \%$ paraformaldehyde, washed extensively, incubated with 2 to $5 \mu \mathrm{g}$ allogeneic IgG per $1 \times 10^{5}$ tumor cells, and then washed to remove excess antibodies. DC activation with the above IgG-IC was repeated in at least 5 independent experiments in biological duplicates.

Phosphatases inhibitor assays. For tumor uptake and mouse DC activation assays, $2.5 \times 10^{5} \mathrm{MoDC}$ and TADC per well were plated on confocal plates and incubated with $1 \times 10^{5} \mathrm{~B} 16$-IC alone or in the presence of $20 \mathrm{nM}$ orthovanadate, a broad tyrosine phosphatase inhibitor, $14 \mathrm{nM}$ PTEN inhibitor bisperoxo oxovanadate dipotassium salt [bpV(HOpic), Millipore], and $43 \mu \mathrm{M}$ Hydroxyphenacyl bromide (Santa Cruz), a SHP-1 inhibitor. Human MoDC were activated with HeLa-IC alone or in the presence of $14 \mathrm{nM}$ bpV(HOpic) (Millipore), a PTEN inhibitor and $43 \mu \mathrm{M}$ 4-Hydroxyphenacyl bromide (Santa Cruz), a SHP-1 inhibitor; 355 nM 7-aza-8-hydroxyquinoline, a SHP-1 and SHP-2 inhibitor (Millipore); or 2.1 $\mu$ M PhenylHydrazonoPyrazolone Sulfonate 1, a SHP-2 inhibitor (Santa Cruz).

For measurement of kinase phosphorylation by FACS, $1 \times 10^{5}$ mouse DC were incubated with $70 \mathrm{nM}$ bpV(HOpic) (Millipore), a PTEN inhibitor, and $355 \mathrm{nM}$ 7-aza-8-hydroxyquinoline, a SHP-1 and SHP-2 inhibitor (Millipore), for 1 hour. Thereafter, $1 \times 10^{5}$ B16 tumor-IC were added to DC cultures for 1 minute and 15 minutes.

In vivo tumor vaccination models. For tumor recurrence studies, $2 \times 10^{5}$ tumor cells were injected s.c. above the right flank, and the size of growing tumors was measured using calipers. When tumors reached 30-40 $\mathrm{mm}^{2}$ for LMP tumors and $16-25 \mathrm{~mm}^{2}$ for B16 tumors, mice were anesthetized and visible mac- 
roscopic tumor was surgically removed. Resected tumors were enzymatically digested with $0.1 \mathrm{mg} / \mathrm{ml}$ DNase I (Sigma-Aldrich) and $5 \mathrm{mg} / \mathrm{ml}$ collagenase IV (Sigma-Aldrich) in HBSS. Cells were then fixed in $2 \%$ paraformaldehyde for 10 minutes, washed extensively in PBS, and coated for 30 minutes with syngeneic or allogeneic antibodies. Antibody-coated tumor cells were then washed and injected into tumor-resected mice $\left(2 \times 10^{6}\right.$ per mouse) or were added to DC cultures. After overnight incubation, DC were washed, and $2.5 \times 10^{6}$ were injected s.c. into tumor-resected mice 1 day after the tumors were removed, adjacent to the site of tumor resection. For prophylactic vaccination assays, MoDC and TADC were incubated overnight with B16 alloIgG-IC, alone or in the presence of PTEN and SHP-1 inhibitors. DC were then washed and $1.6 \times 10^{6}$ cells per mouse were injected s.c. After 5 days $2.5 \times 10^{4} \mathrm{~B} 16$ cells were injected s.c. above the right flank and tumor growth was monitored. At least 3 independent experiments were performed with 4 mice per group. For immunization experiments in an adjuvant setting, $500 \mu \mathrm{g}$ of allogeneic IgG was injected twice, 2 days apart, 1 day after the tumor was resected from the mouse. Experiments were repeated independently at least 3 times with 4 to 5 mice per group.

$T$ cell proliferation. $3 \times 10^{4} \mathrm{DC}$ were cocultured with $3 \times 10^{5}$ MACS-enriched CD8 ${ }^{+}$or $\mathrm{CD} 4^{+} \mathrm{T}$ cells (Miltenyi) from the spleens of LMP- or B16-immunized mice in the presence of 2 IU of IL-2 (Peprotech). After 6 days, cells were pulsed with ${ }^{3} \mathrm{H}$-thymidine $(1 \mu \mathrm{Ci} /$ well $)$ and cultured for an additional 18 hours before being harvested in a Harvester 400 (Tomtec). Radioactivity was measured by a 1450 MicroBeta counter (LKB Wallac). T cell proliferation assays were repeated 5 times with 3 biological replicates and 6 technical replicates for each experimental group.

Immunofluorescence. DC or monocytes were incubated overnight on glass-bottom culture plates (In Vitro Scientific) with CFSE-labeled tumor cells with or without antibodies. Cells were gently washed with PBS (Gibco), fixed for 20 minutes with 2\% paraformaldehyde, and permeabilized with $0.5 \%$ saponin (Sigma-Aldrich). Samples were blocked with 10\% nonimmune goat serum and stained with 1:100 Alexa-conjugated anti-mouse IgG (Invitrogen) and 1:100 anti-mouse I-Ab (BD Biosciences). DC immunostainings were independently repeated at least 3 times in biological duplicates and 3 fields were documented in each slide. Images were collected using a Zeiss 700 confocal laser scanning microscope and analyzed using ZEN software (Carl Zeiss Microscopy).

Immunohistochemistry. Specimens were fixed in $4 \%$ paraformaldehyde, equilibrated in a $20 \%$ sucrose solution, and embedded in frozen tissue matrix (Tissue-Tek OCT). Slides were cut to $5 \mu \mathrm{m}$, blocked with $10 \%$ nonimmune goat serum, and stained with the indicated antibodies. Images were collected using a Zeiss 700 confocal laser scanning microscope and analyzed using ZEN software (Carl Zeiss Microscopy). Tumor immunostaining was repeated independently at least 3 times in biological duplicates, and 3 fields were captured for each slide.

Statistics. Sample size was chosen such that statistical significance could be achieved using appropriate statistical tests (e.g., ANOVA), with errors approximated from previously reported studies. Statistical tests were performed in Prism (GraphPad Software Inc.) to analyze experimental data. In cases in which more than two conditions were compared across groups, statistical significance was determined by 2-way ANOVA with Tukey adjustment for multiple comparisons. In cases in which two conditions were compared across groups, statistical significance was determined by 2-way ANOVA with Sidak's multiple comparisons test. Statistical significance of survival curves was assessed using the log-rank Mantel-Cox test. In all cases, multiple hypothesis testing-adjusted $P$ values were used to assess significance. Phospho-specific flow cytometry data were transformed by taking the inverse hyperbolic sine ( $\operatorname{arcsinh})$, and ratios were taken over the corresponding baseline (unstimulated) value as previously described (69). $P$ values indicate significance of the difference between experimental and control values. $P$ values of less than 0.05 were considered significant.

Study approval. All animal protocols were approved by the Stanford University Institutional Animal Care and Use Committee (Palo Alto, California, USA) under protocol APLAC-17466. The human subjects protocols were approved by Stanford's Institutional Review Board (Palo Alto, California, USA), and informed consent was obtained from all subjects prior to participation in the study.

\section{Author contributions}

YC and TRP designed, conducted, and analyzed experiments and wrote the manuscript. MHS, ILL, NERF, and NB designed and conducted some experiments. MHS analyzed some experiments. JC helped conduct experiments. MHS, ILL, NB, HZ, XZ, PAB, and MNA provided critical feedback in designing 
experiments and in review of the manuscript. BMB recruited patients and provided tissue. EGE supervised the project and contributed to design of the experiment and writing of the manuscript.

\section{Acknowledgments}

Flow cytometry data acquisition and cell sorting were performed at the Stanford Blood Center Flow Cytometry Facility. We thank Lorna Tolentino, Nancy Wu, and Okmi Choi for expert technical support. Research reported in this publication was supported by the Office of the Assistant Secretary of Defense for Health Affairs through the Breast Cancer Research Program under award number W81XWH-15-1-0037; by the National Cancer Institute of the National Institutes of Health under award numbers R01CA196657 (to EGE), F30CA196145 (to TRP), F31CA189331 (to MHS), F31CA196029 (to ILL), and F32CA189408 (to NERF); and by the National Institute of General Medical Sciences of the National Institutes of Health under award number T32GM007365, with additional support from Stanford School of Medicine (to TRP). The content is solely the responsibility of the authors and does not necessarily represent the official views of the National Institutes of Health. Opinions, interpretations, conclusions, and recommendations are those of the authors and are not necessarily endorsed by the Department of Defense.

Address correspondence to: Edgar G. Engleman and Yaron Carmi, 3373 Hillview Avenue, Palo Alto, California 94304, USA. Phone: 650.723.7960; E-mail: edengleman@stanford.edu (E.G. Engleman) and yaron. carmi@gmail.com (Y. Carmi).

1. Hanahan D, Coussens LM. Accessories to the crime: functions of cells recruited to the tumor microenvironment. Cancer Cell. 2012;21(3):309-322.

2. Grivennikov SI, Greten FR, Karin M. Immunity, inflammation, and cancer. Cell. 2010;140(6):883-899.

3. Gajewski TF, Schreiber H, Fu YX. Innate and adaptive immune cells in the tumor microenvironment. Nat Immunol. 2013;14(10):1014-1022.

4. Whiteside TL. The tumor microenvironment and its role in promoting tumor growth. Oncogene. 2008;27(45):5904-5912.

5. Rudensky AY, Gavin M, Zheng Y. FOXP3 and NFAT: partners in tolerance. Cell. 2006;126(2):253-256.

6. Andreu $\mathrm{P}$, et al. FcRgamma activation regulates inflammation-associated squamous carcinogenesis. Cancer Cell. 2010;17(2):121-134.

7. Gabrilovich DI, Ostrand-Rosenberg S, Bronte V. Coordinated regulation of myeloid cells by tumours. Nat Rev Immunol. 2012;12(4):253-268.

8. Mantovani A, Sica A. Macrophages, innate immunity and cancer: balance, tolerance, and diversity. Curr Opin Immunol. 2010;22(2):231-237.

9. Schreiber RD, Old LJ, Smyth MJ. Cancer immunoediting: integrating immunity's roles in cancer suppression and promotion. Science. 2011;331(6024):1565-1570.

10. Coussens LM, Zitvogel L, Palucka AK. Neutralizing tumor-promoting chronic inflammation: a magic bullet? Science. 2013;339(6117):286-291.

11. Galon J, et al. Type, density, and location of immune cells within human colorectal tumors predict clinical outcome. Science 2006;313(5795):1960-1964.

12. Pagès F, et al. Effector memory T cells, early metastasis, and survival in colorectal cancer. N Engl J Med. 2005;353(25):26542666.

13. Fridman WH, Pagès F, Sautès-Fridman C, Galon J. The immune contexture in human tumours: impact on clinical outcome. Nat Rev Cancer. 2012;12(4):298-306.

14. Pardoll DM. The blockade of immune checkpoints in cancer immunotherapy. Nat Rev Cancer. 2012;12(4):252-264.

15. Rosenberg SA, et al. Durable complete responses in heavily pretreated patients with metastatic melanoma using T-cell transfer immunotherapy. Clin Cancer Res. 2011;17(13):4550-4557.

16. Kalos M, et al. T cells with chimeric antigen receptors have potent antitumor effects and can establish memory in patients with advanced leukemia. Sci Transl Med. 2011;3(95):95ra73.

17. Hodi FS, et al. Improved survival with ipilimumab in patients with metastatic melanoma. N Engl J Med. 2010;363(8):711-723.

18. Joffre OP, Segura E, Savina A, Amigorena S. Cross-presentation by dendritic cells. Nat Rev Immunol. 2012;12(8):557-569.

19. Palucka K, Banchereau J. Dendritic-cell-based therapeutic cancer vaccines. Immunity. 2013;39(1):38-48.

20. Melief CJ. Cancer immunotherapy by dendritic cells. Immunity. 2008;29(3):372-383.

21. Steinman RM, Banchereau J. Taking dendritic cells into medicine. Nature. 2007;449(7161):419-426.

22. Carmi Y, et al. Allogeneic IgG combined with dendritic cell stimuli induce antitumour T-cell immunity. Nature. 2015;521(7550):99-104.

23. Nimmerjahn F, Ravetch JV. Fcgamma receptors as regulators of immune responses. Nat Rev Immunol. 2008;8(1):34-47.

24. Vidarsson G, Dekkers G, Rispens T. IgG subclasses and allotypes: from structure to effector functions. Front Immunol. 2014;5:520.

25. Ravetch JV, Bolland S. IgG Fc receptors. Annu Rev Immunol. 2001;19:275-290.

26. Guilliams M, Bruhns P, Saeys Y, Hammad H, Lambrecht BN. The function of Fcy receptors in dendritic cells and macrophages. Nat Rev Immunol. 2014;14(2):94-108. 
27. Nimmerjahn F, Ravetch JV. Divergent immunoglobulin g subclass activity through selective Fc receptor binding. Science. 2005;310(5753):1510-1512.

28. Rafiq K, Bergtold A, Clynes R. Immune complex-mediated antigen presentation induces tumor immunity. J Clin Invest. 2002;110(1):71-79.

29. Schuurhuis DH, et al. Immune complex-loaded dendritic cells are superior to soluble immune complexes as antitumor vaccine. J Immunol. 2006;176(8):4573-4580.

30. Helft J, et al. GM-CSF mouse bone marrow cultures comprise a heterogeneous population of CD11c(+)MHCII(+) macrophages and dendritic cells. Immunity. 2015;42(6):1197-1211.

31. Schulz KR, Danna EA, Krutzik PO, Nolan GP. Single-cell phospho-protein analysis by flow cytometry. Curr Protoc Immunol. 2007; Chapter 8:Unit 8.17.

32. Mustelin T, Vang T, Bottini N. Protein tyrosine phosphatases and the immune response. Nat Rev Immunol. 2005;5(1):43-57.

33. Reth M, Brummer T. Feedback regulation of lymphocyte signalling. Nat Rev Immunol. 2004;4(4):269-277.

34. Heng TS, Painter MW, Immunological Genome Project Consortium. The Immunological Genome Project: networks of gene expression in immune cells. Nat Immunol. 2008;9(10):1091-1094.

35. de Jong JM, et al. Murine Fc receptors for IgG are redundant in facilitating presentation of immune complex derived antigen to CD8+ T cells in vivo. Mol Immunol. 2006;43(13):2045-2050.

36. DiLillo DJ, Ravetch JV. Differential Fc-receptor engagement drives an anti-tumor vaccinal effect. Cell. 2015;161(5):1035-1045

37. Regnault A, et al. Fcgamma receptor-mediated induction of dendritic cell maturation and major histocompatibility complex class I-restricted antigen presentation after immune complex internalization. J Exp Med. 1999;189(2):371-380.

38. Schuurhuis DH, et al. Antigen-antibody immune complexes empower dendritic cells to efficiently prime specific CD8+ CTL responses in vivo. J Immunol. 2002;168(5):2240-2246.

39. Kalergis AM, Ravetch JV. Inducing tumor immunity through the selective engagement of activating Fcgamma receptors on dendritic cells. J Exp Med. 2002;195(12):1653-1659.

40. Sedlik C, et al. A critical role for Syk protein tyrosine kinase in Fc receptor-mediated antigen presentation and induction of dendritic cell maturation. J Immunol. 2003;170(2):846-852.

41. Mócsai A, Ruland J, Tybulewicz VL. The SYK tyrosine kinase: a crucial player in diverse biological functions. Nat Rev Immunol. 2010;10(6):387-402.

42. Minton K. Animal models: unravelling the motheaten phenotype. Nat Rev Immunol. 2013;13(5):306.

43. Abram CL, Roberge GL, Pao LI, Neel BG, Lowell CA. Distinct roles for neutrophils and dendritic cells in inflammation and autoimmunity in motheaten mice. Immunity. 2013;38(3):489-501.

44. Kaneko T, et al. Dendritic cell-specific ablation of the protein tyrosine phosphatase Shp1 promotes Th1 cell differentiation and induces autoimmunity. J Immunol. 2012;188(11):5397-5407.

45. Dhodapkar KM, et al. Selective blockade of inhibitory Fcgamma receptor enables human dendritic cell maturation with IL-12p70 production and immunity to antibody-coated tumor cells. Proc Natl Acad Sci U S A. 2005;102(8):2910-2915.

46. Boross $\mathrm{P}$, et al. The inhibiting Fc receptor for IgG, Fc $\gamma$ RIIB, is a modifier of autoimmune susceptibility. J Immunol. 2011;187(3):1304-1313.

47. Bolland S, Ravetch JV. Spontaneous autoimmune disease in $\mathrm{Fc}$ (gamma)RIIB-deficient mice results from strain-specific epistasis Immunity. 2000;13(2):277-285.

48. Yuasa T, et al. Deletion of fcgamma receptor IIB renders H-2(b) mice susceptible to collagen-induced arthritis. $J$ Exp Med. 1999;189(1):187-194.

49. Ichii O, Konno A, Sasaki N, Endoh D, Hashimoto Y, Kon Y. Altered balance of inhibitory and active Fc gamma receptors in murine autoimmune glomerulonephritis. Kidney Int. 2008;74(3):339-347.

50. Geahlen RL. Getting Syk: spleen tyrosine kinase as a therapeutic target. Trends Pharmacol Sci. 2014;35(8):414-422

51. Krawczyk CM, et al. Toll-like receptor-induced changes in glycolytic metabolism regulate dendritic cell activation. Blood. 2010;115(23):4742-4749.

52. Park D, Lapteva N, Seethammagari M, Slawin KM, Spencer DM. An essential role for Akt1 in dendritic cell function and tumor immunotherapy. Nat Biotechnol. 2006;24(12):1581-1590.

53. Sathaliyawala T, et al. Mammalian target of rapamycin controls dendritic cell development downstream of Flt3 ligand signaling. Immunity. 2010;33(4):597-606.

54. Helgason CD, et al. Targeted disruption of SHIP leads to hemopoietic perturbations, lung pathology, and a shortened life span. Genes Dev. 1998;12(11):1610-1620.

55. Liu Q, et al. SHIP is a negative regulator of growth factor receptor-mediated PKB/Akt activation and myeloid cell survival. Genes Dev. 1999;13(7):786-791.

56. Aich J, Mabalirajan U, Ahmad T, Agrawal A, Ghosh B. Loss-of-function of inositol polyphosphate-4-phosphatase reversibly increases the severity of allergic airway inflammation. Nat Commun. 2012;3:877.

57. Neill L, Tien AH, Rey-Ladino J, Helgason CD. SHIP-deficient mice provide insights into the regulation of dendritic cell development and function. Exp Hematol. 2007;35(4):627-639.

58. Turnquist HR, Raimondi G, Zahorchak AF, Fischer RT, Wang Z, Thomson AW. Rapamycin-conditioned dendritic cells are poor stimulators of allogeneic CD4+ T cells, but enrich for antigen-specific Foxp3+ T regulatory cells and promote organ transplant tolerance. J Immunol. 2007;178(11):7018-7031.

59. Chiang $\mathrm{PH}$, et al. Mechanistic insights into impaired dendritic cell function by rapamycin: inhibition of Jak2/Stat4 signaling pathway. J Immunol. 2004;172(3):1355-1363.

60. Siragam V, Crow AR, Brinc D, Song S, Freedman J, Lazarus AH. Intravenous immunoglobulin ameliorates ITP via activating Fc gamma receptors on dendritic cells. Nat Med. 2006;12(6):688-692.

61. Othy S, et al. Effect of IVIg on human dendritic cell-mediated antigen uptake and presentation: role of lipid accumulation. J Autoimmun. 2012;39(3):168-172.

62. Bayry J, et al. Inhibition of maturation and function of dendritic cells by intravenous immunoglobulin. Blood. 2003;101(2):758-765. 
63. Mosser DM, Edwards JP. Exploring the full spectrum of macrophage activation. Nat Rev Immunol. 2008;8(12):958-969.

64. de Visser KE, Korets LV, Coussens LM. De novo carcinogenesis promoted by chronic inflammation is B lymphocyte dependent. Cancer Cell. 2005;7(5):411-423.

65. Affara NI, et al. B cells regulate macrophage phenotype and response to chemotherapy in squamous carcinomas. Cancer Cell. 2014;25(6):809-821.

66. Mahnke K, Schmitt E, Bonifaz L, Enk AH, Jonuleit H. Immature, but not inactive: the tolerogenic function of immature dendritic cells. Immunol Cell Biol. 2002;80(5):477-483.

67. Steinman RM, Hawiger D, Nussenzweig MC. Tolerogenic dendritic cells. Annu Rev Immunol. 2003;21:685-711.

68. Tseng WW, et al. Development of an orthotopic model of invasive pancreatic cancer in an immunocompetent murine host. Clin Cancer Res. 2010;16(14):3684-3695.

69. Irish JM, et al. B-cell signaling networks reveal a negative prognostic human lymphoma cell subset that emerges during tumor progression. Proc Natl Acad Sci U S A. 2010;107(29):12747-12754. 